Merging Operators: beyond the finite case

José Luis Chacón and Ramón Pino Pérez

Notas de Matemática

Serie: Pre-Print

No. 235

Mérida - Venezuela

2004 


\title{
Merging Operators: beyond the finite case
}

\author{
José Luis Chacón and Ramón Pino Pérez
}

\begin{abstract}
We extend the results of Konieczny and Pino Pérez (J. Log. and Comp. 2002) concerning merging operators in a finite logical framework to the infinite case (countably many propositional variables). The number of sources we consider remains finite. The main result is the representation theorem. In order to prove it, we state some results which are interesting for their own sake. Some postulates had to be restated in a new form. The new form is equivalent to the old one only in the finite case, but more appropriate to deal with the infinite case. The construction of merging operators starting from distances between valuations is also generalized. Indeed, we introduce a new kind of operators built upon the so called Cantor distance.
\end{abstract}

Keywords: Merging operators, Belief revision, Integrity constraints, syncretic assignment

\section{Introduction}

The general problem of merging information is to extract a coherent common information from several sources of information. The most natural thing to do, which consists in taking the sum of pieces of information does not work in general because two (or more) sources of information may be contradictory. In this case, their union will be necessarily contradictory.

A lot of methods have been introduced to merge information in a logical framework $[4,5,2$, 19, 23, 10]. Different sets of logical properties which have to be satisfied by belief merging operators, have been proposed [23, 18, 19, 11, 12]. These works offer a classification of (logical) merging operators. In fact, they give some interesting results concerning a semantic characterization of operators satisfying some postulates of rationality. These results are known as Representation Theorems. Their basic framework is the finite Propositional Logic. Since then, nothing has been done to extend these results to infinite logical framework, i.e. Propositional Logic in which the formulas are built upon a countable set of propositional variables. This is what we do in this work.

The motivations to study the case in which we have countably many propositional variables -the infinite case- are given now. First of all, in the logical framework used to represent a piece of information, the propositional variables play an essential role: they represent the factual information. For instance, facts like the device is broken, inflation has been stopped, etc., are traditionally represented by propositional variables. However, to assume that the number of propositional variables is finite, supposes modeling a situation in which there is no room for new facts. In some 
cases it is interesting to have the possibility of introducing new facts. For instance, when merging information about the causes of bad performance of a microchip, it can be useful to introduce the fact that the intensity of the current is always changing, a new variable not considered up to now. Another situation in which it is very common to introduce new variables, concerns the arguments about the acceptance or rejection of an article for a conference, a journal, etc. In many cases, one source -a reviewer- presents a new argument that can be decisive in the final appreciation.

Thus, one way to open the possibility of considering variables not considered until the present is to have countably many variables from the beginning. One can attach more importance to the first variables. One can imagine the importance attached to a variable to be smaller as the subscript of the variable is bigger. This kind of situation will be captured with some operators built upon a distance called Cantor distance (see Section 3).

Also, in order to introduce the fact that one source of information can carry out infinite information, we have to allow infinite propositional variables. This does not mean that a source has to give always an infinite list of facts or more complex pieces of information. We know that infinite information can be sometimes encoded by finite information. For instance, the theory generated by a finite set of formulas is a truly infinite object which is completely encoded by a finite number of finite objects. The approach used in this work will be to represent the piece of information of an agent (a source) by a logical theory, i.e. a set of propositional formulas closed by logical deduction. Of course, the set of propositional variables will be infinite.

The kind of representation of information used here -theories- is not new in the area of dynamical reasoning. For instance, in Belief Revision $([1,8])$, the epistemic states are represented by logical theories. This kind of representation is quite natural and rich enough. Nevertheless, this is not the richest representation of epistemic states as can be seen in the works of Konieczny, Pino Pérez and Benferhat et al. ([10, 13, 14, 3]). However, in order to deal with the infinite case, the representation of information by a logical theory will give us sufficient insight to treat the problems about postulates and to find a general technique for dealing with the representation theorems.

There is another dimension that can be considered in order to introduce the infinite in the framework of merging: it concerns the number of sources of information. This issue is not addressed in this work. Many are the the reasons for not considering this dimension. The main reason is that, although we think this problem is very interesting from a theoretical point of view, we do not have a very clear idea, at the present time, about the meaning of well behaved operators in this case. Even the meaning of majority in this context is unclear (for a discussion about some problems concerning this issues see [20]). Another reason is that in the most common situations the number of sources of information is finite. However, this number is not fixed before the beginning of the operations. So, we may think that, concerning the number of sources of information, merging information is an operation potentially infinite. It is important to notice that the nature of the operators can totally change if we impose a bound to the number of sources of information (see [16]). Thus, for us, this is the natural way in which the infinite can 
be introduced in this dimension: the number of sources can be arbitrarily big. This will be the case of our operators and it is the case of operators considered in [15].

The representation theorems proved here are, roughly speaking, of the following kind (for more precision see Theorem 4):

A merging operator is represented by an assignment which maps each group of sources of information to a total pre-order over valuations. The result of merging the group under the constraints will be the theory of the minimal models of the constraints (minimal with respect to the pre-order associated to the group).

The difficult part in this kind of theorem is, on one hand, to find the definition of the assignment and, on the other hand, to prove that the definition is indeed adequate. We introduce here a general technique to define this kind of assignments (see Definition 5). We will prove that the definition can be stated in different equivalent ways, each of them useful to prove some properties. All this is inspired by a similar technique used to prove representation theorems for nonmonotonic relations introduced in [21].

This work is organized as follows: in the first Section we present the definitions of merging operators, quasi-merging operators, majority merging operators and arbitration operators. We define also their corresponding notions in terms of assignments: syncretic assignments, quasisyncretic assignments, majority syncretic assignments and arbitration assignments. For each family of operators defined we state a representation theorem. In Section 2 we define some general families of operators starting from a distance (actually pseudo-distance, see Definition 12). We define the family of $\Sigma$-operators which turns out to be a family of majority merging operators. We define the family of Max-operators which turn out to be a family of quasi-merging operators. We define the family of Gmax-operators which turn out to be a family of arbitration merging operators. Next, we find Section 3 which is devoted to giving concrete constructions of the operators defined in the previous Section, using three distances: generalized Dalal distance, discrete distance and Cantor distance. We give some examples of these operators at work. Before an Appendix with technical results and proofs, we have Section 4 with some final remarks and some questions. For the sake of readability, we have put the proofs in the Appendix. However, the Appendix contains some new results, not stated in previous sections, and a deep study of relationships between logical postulates of operators and conditions satisfied by assignments.

\section{Merging operators and representation theorems}

In this section we define merging operators adapting the postulates in [15]. We define the notion of the syncretic assignment needed to state the representation theorems. This is also a modification of a similar notion defined in [15] in the finite case. We end the section stating the representation theorems.

Our logical framework is the infinite propositional calculus. The set of valuations will be denoted by $\mathcal{V}$. When $A$ is a subset of $\mathcal{V}$, we will denote $T h(A)$, the theory of $A$, the set 
$\{\varphi \in \mathcal{L}: \forall M \in A, M \models \varphi\}$. Let $\mathcal{T}$ be the set of all the theories. Let $\mathcal{T}^{*}$ be the set of consistent theories. Let $\mathcal{S}=\mathcal{M}\left(\mathcal{T}^{*}\right)$ where $\mathcal{M}\left(\mathcal{T}^{*}\right)$ is the set of finite multi-sets with elements in $\mathcal{T}^{*}$. The elements of $\mathcal{S}$ will be denoted with uppercase Greek letters $\Phi, \Psi, \ldots$ (eventually with subscripts); they are called knowledge multi-sets ${ }^{1}$, while the elements of $\mathcal{T}$ are called knowledge bases. We use the notation $K, H, R, S, T$ (eventually with subscripts) for the theories. We denote by $\bmod (K)$ the set of models of $K$, i.e. the set $\{M \in \mathcal{V}: M \models K\}$. We denote $\cup \Phi=\{\alpha: \exists K \in \Phi$ such that $\alpha \in K\}$, and $\mathbb{M} \Phi=\operatorname{Cn}(\cup \Phi)$, where $\mathrm{Cn}_{\mathrm{n}}$ is the operator of classical consequences. The union of multi-sets is denoted by the symbol $\sqcup$. For convenience, we write $K \sqcup T$ instead of $\{K\} \sqcup\{T\}$, and $K^{n}$ for the multi-set $\{K, K \ldots K\}$ where $K$ appears $n$ times. Similarly for $\Phi$ a knowledge multi-set we define $\Phi^{n}$ by induction as follows: $\Phi^{1}=\Phi$ and $\Phi^{n+1}=\Phi^{n} \sqcup \Phi$.

The candidates to be merging operators are applications of the following form

$$
\Delta: \mathcal{S} \times \mathcal{T} \longrightarrow \mathcal{T}
$$

$\Delta(\Phi, K)$ will be denoted by $\Delta_{K}(\Phi) . \quad K$ represents the integrity constraints. Such applications will be called operators. Such an operator $\Delta$ is said to be a merging operator if the following postulates hold:

(IC0)

(IC1)

(IC2)

(IC3)

(IC4)

(IC5)

(IC6)

(IC7)

$$
\Delta_{K}(\Phi) \supseteq K
$$

$$
K \nvdash \perp \Rightarrow \Delta_{K}(\Phi) \nvdash \perp
$$

$$
K \cup(\mathbb{A} \Phi) \nvdash \perp \quad \Rightarrow \quad \Delta_{K}(\Phi)=\operatorname{Cn}(K \cup(\mathbb{A} \Phi))
$$$$
\left[T \subseteq K \wedge T \subseteq K^{\prime} \wedge \Delta_{T}\left(K \sqcup K^{\prime}\right) \cup K \nvdash \perp\right] \Rightarrow \Delta_{T}\left(K \sqcup K^{\prime}\right) \cup K^{\prime} \nvdash \perp
$$

$$
\operatorname{Cn}\left(\Delta_{K}\left(\Phi_{1}\right) \cup \Delta_{K}\left(\Phi_{2}\right)\right) \supseteq \Delta_{K}\left(\Phi_{1} \sqcup \Phi_{2}\right)
$$

$$
\Delta_{K}\left(\Phi_{1}\right) \cup \Delta_{K}\left(\Phi_{2}\right) \nvdash \perp \Rightarrow \Delta_{K}\left(\Phi_{1} \sqcup \Phi_{2}\right) \supseteq \operatorname{Cn}\left(\Delta_{K}\left(\Phi_{1}\right) \cup \Delta_{K}\left(\Phi_{2}\right)\right)
$$$$
\mathrm{Cn}\left(\Delta_{K_{1}}(\Phi) \cup K_{2}\right) \supseteq \Delta_{\mathrm{Cn}\left(K_{1} \cup K_{2}\right)}(\Phi)
$$

$$
\Delta_{K_{1}}(\Phi) \cup K_{2} \nvdash \perp \Rightarrow \Delta_{\mathrm{Cn}\left(K_{1} \cup K_{2}\right)}(\Phi) \supseteq \operatorname{Cn}\left(\Delta_{K_{1}}(\Phi) \cup K_{2}\right)
$$

The first postulate, ( $\mathrm{IC} 0)$, is the postulate of persistence of constraints: in presence of a set of constraints $K$, the result of merging information has to contain the constraints. This is appropriate when the theory $K$ represents a piece of information that only can be enriched by adding information, i.e. the information contained in $K$ have to remain. Note that the instanciation of postulates to the case in which the constraints are the set of tautologies, simulates pure merging, that is, merging without integrity constrains. The second postulate, (IC1), is the postulate of consistency: it requires that the result of merging is always consistent unless the constraints impose the contrary. The third postulate, (IC2), is the postulate of minimality: if the union of pieces of information together with the constraints is a coherent piece of information, then the result of merging is the smallest theory containing this last piece of information. The

\footnotetext{
${ }^{1}$ This corresponds to the notion of knowledge set in [15]. Here we have change a little the name to avoid confusion with the notion of knowledge set comming from Belief Revision.
} 
fourth postulate, (IC3), is the fairness postulate: if two sources of information carry out the information contained in the constraints (eventually more) then the result of merging has to be fair, in the sense that if the result is consistent with one source it has to be consistent with the other one. The following two postulates deal with the behavior of the groups of sources of information and its subgroups. The fifth postulate, (IC4), is the postulate of coherence of groups: suppose that we make a partition of a group of sources of information in two subgroups, then the result of merging the whole group of sources of information has to be contained in the smallest theory which contains the result of merging the subgroups. The sixth postulate, (IC5), is the postulate of strong coherence of groups: suppose that we make a partition of a group of sources of information in two subgroups and that the result of putting together the merging of the subgroups is a consistent set, then the result of merging the whole group contains this consistent set. Note that (IC4) and (IC5) together say that when the result of putting together the merging of the subgroups is a consistent set, then the result of merging the whole group is exactly the smallest theory containing this consistent set. The last two postulates concern the (iterative) behavior of integrity constraints. The seventh postulate, (IC6), tells us that the result of merging the information under a set of integrity constraints enriched by a new set of integrity constraints is contained in the smallest theory containing the new set of integrity constraints and the merging under the old set of integrity constraints. The last postulate, (IC7), says that if the smallest theory containing the new set of integrity constraints and the merging under the old set of integrity constraints is consistent, then it is contained in the result of merging the information under a set of integrity constraints formed by the old integrity constraints plus the new set of integrity constraints. The postulates (IC6) and (IC7) are a generalization of postulates $\left(\mathrm{K}^{*} 7\right)$ and $\left(\mathrm{K}^{*} 8\right)$ in the framework of belief revision (see [8]).

\section{Remark 1}

Remember that in the finite case, in which formulas are used to represent a piece of information, the postulate expressing the independence of syntax have full meaning: operators have to be invariant under logical equivalence between formulas and an equivalence between the knowledge multi-sets. In the current framework there is no postulate corresponding to independence of syntax because it would be trivial. Actually, two theories are equivalent if and only if they are identical. Thus, in our framework, the postulate corresponding to independence of syntax is tautological.

However, there is a correspondance one-to-one between the postulates presented here and those presented in [12, 15]. This correspondance is stated as follows: for $i=0,1,2$ the postulate IC $i$ here corresponds to postulate IC $i$ there. For $i=3,4,5,6,7$ the postulate IC $i$ here corresponds to postulate $I C i+1$ there. Thus, there is a shift in the enumeration of the postulates with respect to the works mentioned above.

Next, we are interested in studying mappings (assignments) from $\mathcal{S}$ into the collection of total pre-orders over valuations (i.e. reflexive and transitive relations which are total). Some of these mappings will be enough to represent our merging operators.

Let us recall some general standard notations concernig a total pre-order $\leq$ over a set $X$. The relation $\sim$ associated to $\leq$ is defined by $x \sim y$ iff $x \leq y$ and $y \leq x$. The relation $\sim$ is an equivalence relation. The relation $<$ associated to $\leq$ is defined by $x<y$ iff $x \leq y$ and $y \not \leq x$. The relation $<$ is transitive, irreflexive and asymmetric. 
In order to define the good assignments we need to introduce explicitly a property which is trivially satisfied in the finite case. This kind of property has been introduced by Kraus et al. in [17] in order to have representation theorems for Nonmonotonic relations.

\section{Definition 2}

A total pre-order $\leq_{\Phi}$ over $\mathcal{V}$ is said to be smooth if for all $K \in \mathcal{T}$ and for all $M \models K$ that is not minimal in $\bmod (K)$, there exists $N=K$ such that $N$ is minimal in $\bmod (K)$ and $N<_{\Phi} M$.

Notice that, for a total pre-order $\leq_{\Phi}$ the smoothness condition can be expressed by the following

$$
\forall K \in \mathcal{T}^{*}, \min \left(\bmod (K), \leq_{\Phi}\right) \neq \varnothing
$$

because, by the totality, for any $M \in \bmod (K)$ and $N \in \min \left(\bmod (K), \leq_{\Phi}\right)$ we have $N \leq_{\Phi}$.

Notice that a well founded relation is smooth but the converse is not true. For a discussion about this see [17].

\section{Definition 3}

A syncretic assignment is a mapping $\left(\Phi \mapsto \leq_{\Phi}\right)$ that assigns to each knowledge multi-set $\Phi \in \mathcal{S}$ a total pre-order $\leq_{\Phi}$ over the set of valuations $\mathcal{V}$ verifying the following conditions:
1) If $M \models \mathbb{A} \Phi, N \models \mathbb{A} \Phi$, then $M \simeq_{\Phi} N$
2) If $M \models \mathbb{A} \Phi, N \not \models \mathbb{A} \Phi$, then $M<_{\Phi} N$
3) $\forall M \models K \quad \exists N \models T \quad$ such that $\quad N \leq_{K \sqcup T} M$
4) If $M \leq_{\Phi_{1}} N$ and $M \leq_{\Phi_{2}} N$, then $M \leq_{\Phi_{1} \sqcup \Phi_{2}} N$
5) If $M<_{\Phi_{1}} N$ and $M \leq_{\Phi_{2}} N$, then $M<_{\Phi_{1} \sqcup \Phi_{2}} N$
6) $\leq_{\Phi}$ is smooth

The link between these assignments and merging operators is showed in the next (representation) theorem:

\section{Theorem 4}

An application $\Delta: \mathcal{S} \times \mathcal{T} \longrightarrow \mathcal{T}$ is a merging operator if and only if there exists a syncretic assignment $\Phi \mapsto \leq_{\Phi}$ such that

$$
\Delta_{K}(\Phi)=\operatorname{Th}\left(\min \left(\bmod (K), \leq_{\Phi}\right)\right)
$$

Given a syncretic assignment, it is quite straightforward to see that the operator defined by the previous equality verifies the postulates of merging operator. The converse is not so straightforward. The crucial point is the definition of the total pre-order $\leq_{\Phi}$ associated to $\Phi$ when we have a merging operator $\Delta$. The next definition, inspired by Pino Pérez and Uzcátegui's work about representation of nonmonotonic relations [21], tells us how to proceed. 


\section{Definition 5}

Let $\Delta$ be a mapping of $\mathcal{S} \times \mathcal{T}$ into $\mathcal{T}$. For any $\Phi \in \mathcal{S}$ we define a relation $\leq_{\Phi}$ over $\mathcal{V}$ by putting

$$
M \leq_{\Phi} N \stackrel{\text { def }}{\Longleftrightarrow} \forall K, T \in \mathcal{T}\left[M \models \Delta_{K}(\Phi) \wedge N \models \Delta_{T}(\Phi) \rightarrow M \models \Delta_{K \cap T}(\Phi)\right]
$$

Notice that $K \cap T$ is a theory if $K$ and $T$ are theories. Thus in the previous definition we do not need to put $\operatorname{Cn}(K \cap T)$.

Most of the work will consist in showing that this relation is a total pre-order when $\Delta$ is a merging operator and that in fact this pre-order represents the operator, i.e. the equation 1 holds.

There are other classes of natural operators that can be characterized in terms of some family of assignments. First define the following postulate:

$$
\left(\text { IC5 }^{\prime}\right) \quad \Delta_{K}\left(\Phi_{1}\right) \cup \Delta_{K}\left(\Phi_{2}\right) \not \perp \perp \quad \Rightarrow \quad \Delta_{K}\left(\Phi_{1} \sqcup \Phi_{2}\right) \supseteq \Delta_{K}\left(\Phi_{1}\right) \cap \Delta_{K}\left(\Phi_{2}\right)
$$

A operator $\Delta$ is said to be quasi-merging operator if the postulates (IC0)-(IC4),(IC5'),(IC6),(IC7) hold.

The counterpart in terms of assignments is as follows. An assignment $\Phi \mapsto \leq_{\Phi}$, where $\leq_{\Phi}$ is a total pre-order over $\mathcal{V}$, is said to be a quasi-syncretic assignment if it satisfies the conditions $1-4$ and 6 given in Definition 3 plus the following:

$$
\left.5^{\prime}\right) \quad \text { If } M<_{\Phi_{1}} N \text { and } M<_{\Phi_{2}} N \text {, then } M<_{\Phi_{1} \sqcup \Phi_{2}} N
$$

Now, we can state our second representation theorem:

\section{Theorem 6}

An operator $\Delta$ is a quasi-merging operator if and only if there exists a quasi-syncretic assignment $\Phi \mapsto \leq_{\Phi}$ such that

$$
\Delta_{K}(\Phi)=\operatorname{Th}\left(\min \left(\bmod (K), \leq_{\Phi}\right)\right)
$$

\section{Remark 7}

In semantical terms (IC5) says that the set of models of $\Delta_{K}\left(\Phi_{1} \sqcup \Phi_{2}\right)$ is contained in the intersection of the set models of $\Delta_{K}\left(\Phi_{1}\right)$ and the set of models of $\Delta_{K}\left(\Phi_{2}\right)$. On the other hand, (IC5') says that the models of $\Delta_{K}\left(\Phi_{1} \sqcup \Phi_{2}\right)$ is contained in the union of the set of models of $\Delta_{K}\left(\Phi_{1}\right)$ and the set of models of $\Delta_{K}\left(\Phi_{2}\right)$. It is interesting to point out is that this difference between these two postulates is reduced to an apparently little difference in terms of assignments. Thus, this little difference between the condition 5 and the condition 5' is indeed significant.

\section{Two classes of merging operators: Majority and arbitration}

We define in the rest of this section two classes of merging operators. The first one is the class of majority merging operators. The operators in this class are supposed to give account of some 
majority behavior in extracting information from several sources: if many sources give us a piece of information, this piece of information will persist in the result of merging.

The second class introduced is the class of arbitration merging operators. The operators in this class are supposed to give account of some consensual behavior in extracting information from several sources: a piece of information that is unlikely for one source of information will have a tendency to be rejected in the result of merging. Conversely, a piece of information that is likely for all the sources will have a tendency to remain in the result after merging.

We define also two classes of assignments corresponding exactly to these classes of operators. For each class we state the corresponding representation theorem.

A merging operator is said to be a majority merging operator iff the following postulate holds

(Maj) $\forall \Phi_{1}, \forall \Phi_{2}, \exists n$ such that $\forall K \in \mathcal{T}, \Delta_{K}\left(\Phi_{1} \sqcup \Phi_{2}{ }^{n}\right) \supseteq \Delta_{K}\left(\Phi_{2}\right)$.

We can paraphrase this postulate in the following way when $\Phi_{2}$ is a singleton: if in the multiple observation $\Phi_{1} \sqcup \Phi_{2}{ }^{n}$ the belief (the observation) $\Phi_{2}$ is repeated enough ( $n$ times, $n$ depending upon $\Phi_{1}$ and $\Phi_{2}$ ), then the result will contain the information resulting of observing only $\Phi_{2}$. Note that the postulate do not say which is the size of $n$ in terms of the cardinalities of the belief sets $\Phi_{1}$ and $\Phi_{2}$. Remember than in terms of Voting Theory an operator of choice has (strictly) majoritary behavior if when it is applied to a profile in which a candidate have more than the half of votes, he is choosen. So, we can see the postulate (Maj) as generalization of this kind of behaviors.

Note that in this new version of (Maj) we write explicitely the quantifiers in order to avoid any confusion. The version in [15] states

$$
\exists n \quad \Delta_{K}\left(\Phi_{1} \sqcup \Phi_{2}^{n}\right) \supseteq \Delta_{K}\left(\Phi_{2}\right)
$$

that, with the usual conventions about quantifiers, means

$$
\forall \Phi_{1}, \forall \Phi_{2}, \forall K \in \mathcal{T}, \exists n \Delta_{K}\left(\Phi_{1} \sqcup \Phi_{2}{ }^{n}\right) \supseteq \Delta_{K}\left(\Phi_{2}\right)
$$

The version of (Maj) in this paper is clearly stronger than the correspondent postulate in [15]. Indeed, in the finite case they are equivalent. We conjecture that in the infinite case the current version of (Maj) does not follow from 2 above.

A syncretic assignment is majority syncretic assignment iff the following condition holds:

$$
\text { 7) If } M<_{\Phi_{2}} N \text {, then } \exists n \quad M<_{\Phi_{1} \sqcup \Phi_{2}{ }^{n} N}
$$

\section{Theorem 8}


An operator $\Delta$ is a majority merging operator iff there exists a majority syncretic assignment such that

$$
\Delta_{K}(\Phi)=\operatorname{Th}\left(\min \left(\bmod (K), \leq_{\Phi}\right)\right)
$$

In order to establish the postulate characterizing the merging operators having a consensual behavior we need to introduce some concepts and notation. Let $A$ and $B$ be two sets, we denote $A \triangle B$ their symmetrical difference, i.e. $A \triangle B=(A \backslash B) \cup(B \backslash A)$ where $A \backslash B=\{x \in A: x \notin B\}$. Let $K$ and $T$ be two theories, their symmetrical difference, denoted $K \diamond T$ is defined as follows:

$$
K \diamond T=T h(\bmod (K) \triangle \bmod (T)) .
$$

A merging operator is said to be an arbitration merging operator if the following postulate holds:

$$
\left.\begin{array}{l}
K_{1} \nsubseteq K_{2}, \quad K_{2} \nsubseteq K_{1}, \\
(\text { Arb }) \quad \Delta_{K_{1}}\left(H_{1}\right)=\Delta_{K_{2}}\left(H_{2}\right), \\
\Delta_{K_{1} \diamond K_{2}}\left(H_{1} \sqcup H_{2}\right)=K_{1} \diamond K_{2}
\end{array}\right\} \Rightarrow \Delta_{K_{1} \cap K_{2}}\left(H_{1} \sqcup H_{2}\right)=\Delta_{K_{1}}\left(H_{1}\right)
$$

This postulate ensures that this is the median possible choices that are preferred. It is much more intuitive when it is expressed in terms of syncretic assignment ( $c$ f.condition 8 below). We will illustrate this on the following scenario $^{2}$ :

\section{Example 9}

Tom and David missed the soccer match yesterday between reds and yellows. So they don't know the result of the match. Tom listened in the morning that reds made a very good match. So he thinks that a win of reds is more plausible than a draw and that a draw is more reliable than a win of yellows. David was told that after that match yellows have now a lot of chances of winning the championship. From this information he infers that yellows won the match, or otherwise at least took a draw. Confronting their points of view, Tom and David agree on the fact that the two teams are of the same strength, and that they had the same chances of winning the match. What arbitration demand is that, with those informations, Tom and David have to agree that a draw between the two teams is the more plausible result.

A syncretic assignment $\Phi \mapsto \leq_{\Phi}$ is said to be an arbitration syncretic assignment if the following condition holds

$$
\text { 8) If } M<_{H_{1}} N, M<_{H_{2}} P, N \simeq_{H_{1} \sqcup H_{2}} P \quad \text { then } \quad M<_{H_{1} \sqcup H_{2}} N \text {. }
$$

\section{Theorem 10}

An operator $\Delta$ is an arbitration merging operator if and only if there exists an arbitration syncretic assignment $\Phi \mapsto \leq_{\Phi}$ such that

$$
\Delta_{K}(\Phi)=\operatorname{Th}\left(\min \left(\bmod (K), \leq_{\Phi}\right)\right) .
$$

\footnotetext{
${ }^{2}$ This example is taken from [16].
} 


\section{Remark 11}

We would like finish this section with a little comment about the scope of theorems 4, 6, 8 and 10, the representation theorems. To build assignments having the required properties of these theorems is a task less hard in our view that the direct construction of operators and the verification of postulates. So, the representation theorem give us a power tool for building and studying these operators. This will be illustrated in sections 2 and 3.

\section{General construction of merging operators}

In this section we consider three general methods to build merging operators from (pseudo) distances between valuations (see definition below). In fact we give methods to construct syncretic assignments and then we use the representation theorems to obtain the operators.

Let $\mathbb{R}^{+}$be the set $\{x \in \mathbb{R}: x \geq 0\}$ and $\overline{\mathbb{R}^{+}}=\mathbb{R}^{+} \cup\{\infty\}$ with the usual order $\leq$ over elements of $\mathbb{R}$ and putting $x \leq \infty$, for any $x \in \mathbb{R}^{+}$. Remember that for any set $A \subseteq \mathbb{R}^{+}$which is non empty, $\inf (A)$ denotes the greatest lower bound of $A$. It is easy to see that the function inf defined over the non empty subsets of $\overline{\mathbb{R}^{+}}$have the following behavior

$$
\inf (B)= \begin{cases}\inf \left(B \cap \mathbb{R}^{+}\right) & \text {if } B \cap \mathbb{R}^{+} \neq \emptyset \\ \infty & \text { otherwise }\end{cases}
$$

\section{Definition 12}

A function $d: \mathcal{V} \times \mathcal{V} \rightarrow \overline{\mathbb{R}^{+}}$is said to be a pseudo-distance if the following conditions hold:

1. $d(M, N)=d(N, M)$.

2. $d(M, N)=0$ iff $M=N$.

3. If $K$ and $T$ are consistent theories, there are $M \models T$ and $N \models K$ such that $d(M, N)=$ $\inf \{d(Q, P): Q \models T, P \models K\}$.

We can extend $d$ to a function $\bar{d}: \mathcal{V} \times \mathcal{T} \rightarrow \overline{\mathbb{R}^{+}}$in the following way:

$$
\bar{d}(M, K)=\inf _{N \mid=K} d(M, N)
$$

In turn we extend the function $\bar{d}$ to a function $\overline{\bar{d}}: \mathcal{T} \times \mathcal{T} \rightarrow \overline{\mathbb{R}^{+}}$as follows

$$
\overline{\bar{d}}(K, S)=\inf _{N=K} \bar{d}(N, S)
$$

By an abuse of notation we will write $d$ instead of $\bar{d}$ and instead of $\overline{\bar{d}}$. It is easy to see that

$$
d(K, S)=\inf _{N \models K, M \models S} d(M, N)=\inf _{M \models S} d(M, K)
$$

Notice that, by the condition 3 of the definition of pseudo-distance, the inf above is indeed a minimum, i.e. it is realized by an element $d(M, N)$ with $M \models K$ and $N \models S$. 


\section{$\Sigma$ and Max operators}

Let $d$ be a pseudo-distance as defined above; we define $d_{\Sigma}: \mathcal{V} \times \mathcal{S} \rightarrow \overline{\mathbb{R}^{+}}$and $d_{\text {max }}: \mathcal{V} \times \mathcal{S} \rightarrow \overline{\mathbb{R}^{+}}$ as follows:

$$
\begin{aligned}
& d_{\Sigma}(M, \Phi)=\sum_{K \in \Phi} d(M, K) \\
& d_{\max }(M, \Phi)=\max \{d(M, K): K \in \Phi\}
\end{aligned}
$$

where, of course, we use the natural extension of + to $\overline{\mathbb{R}^{+}}$, i.e. $r+\infty=\infty+r=\infty$.

Now for each $\Phi$ we define two relations $\leq_{\Phi}^{\Sigma}$ and $\leq_{\Phi}^{\max }$ over $\mathcal{V}$ as follows:

$$
\begin{aligned}
& M \leq_{\Phi}^{\Sigma} N \quad \text { iff } \quad d_{\Sigma}(M, \Phi) \leq d_{\Sigma}(N, \Phi) \\
& M \leq_{\Phi}^{\max } N \quad \text { iff } \quad d_{\max }(M, \Phi) \leq d_{\max }(N, \Phi)
\end{aligned}
$$

\section{Proposition 13}

(i) $\Phi \mapsto \leq_{\Phi}^{\Sigma}$ is a majority syncretic assignment.

(ii) $\Phi \mapsto \leq \leq_{\Phi}^{\max }$ is a quasi-syncretic assignment.

As a corollary of the previous Proposition and Theorems 6 and 8 we have the following result

\section{Corollary 14}

(i) The operator $\Delta^{\Sigma}: \mathcal{S} \times \mathcal{T} \rightarrow \mathcal{T}$ defined by

$$
\Delta_{R}^{\Sigma}(\Phi)=\operatorname{Th}\left(\min \left(\bmod (R), \leq_{\Phi}^{\Sigma}\right)\right)
$$

is a majority merging operator.

(ii) The operator $\Delta^{\max }: \mathcal{S} \times \mathcal{T} \rightarrow \mathcal{T}$ defined by

$$
\Delta_{R}^{\max }(\Phi)=\operatorname{Th}\left(\min \left(\bmod (R), \leq_{\Phi}^{\max }\right)\right)
$$

is a quasi-merging operator.

There is an interesting property dealing with the iterative behavior of an operator that is satisfied by $\Delta^{\Sigma}$ and $\Delta^{\max }$ when $d$ is indeed a distance, i.e. when $d$ satisfies the Triangle Inequality $(d(x, y) \leq d(x, z)+d(z, y))$. To be more precise let us define first the so called iteration property.

Let $R$ and Tbe knowledge bases, $\Phi$ a knowledge multi-set and $\Delta$ an operator, we define the sequence $\left(\Delta_{R}^{n}(\Phi, T)\right)_{n \geq 1}$ in the following way:

$$
\begin{array}{ll}
\text { 1) } & \Delta_{R}^{1}(\Phi, T)=\Delta_{R}(\Phi \sqcup T), \\
\text { 2) } & \Delta_{R}^{n+1}(\Phi, T)=\Delta_{R}\left(\Delta_{R}^{n}(\Phi, T) \sqcup T\right)
\end{array}
$$

The following property is called the iteration property

$$
\text { (ICit) } \quad \text { If } \quad T \supseteq R \text { then } \exists n \Delta_{R}^{n}(\Phi, T) \supseteq T
$$

The intuitive meaning of this property is the following: the result of iterating the operator a number of times bigger enough with respect the same information $T$ will be reach the information $T$. In other words, if the information $T$ is repeated along the time, the operator will go approching to $T$ until to rech it. We think that is a good and natural property of operators. 


\section{Theorem 15}

If the pseudo-distance $d: \mathcal{V} \times \mathcal{V} \rightarrow \overline{\mathbb{R}^{+}}$satisfies the Triangle Inequality, i.e. $d(M, N) \leq d(M, P)+$ $d(P, N)$, then $\Delta^{\Sigma}$ and $\Delta^{\max }$ satisfy (ICit).

We do not know if the converse of the previous theorem holds. More generally, we do not know how to characterize the operators defined via a distance or pseudo-distance.

\section{Gmax operators}

Starting from a pseudo-distance $d$ we are going to build an arbitration syncretic assignment which induces, via the representation theorem 10, an arbitration merging operator that is actually a refinement of the operator $\Delta^{\max }$. The operator Gmax that we are going to define is usually known in Decision Theory as the leximax operator.

\section{Definition 16}

Let $\Phi=\left\{K_{1}, K_{2}, \ldots K_{n}\right\}$ be a knowledge multi-set. For any valuation $M$ we put $\left(d_{1}^{M}, d_{2}^{M} \ldots d_{n}^{M}\right)$ where $d_{i}^{M}=d\left(M, K_{i}\right)$, for $i=1, \ldots, n$. Let $L_{M}^{\Phi}$ be the list $\left(d_{1}^{M}, d_{2}^{M} \ldots d_{n}^{M}\right)$ ordered decreasingly. Let $\leq_{l e x}$ be the lexicographical order between lists. Finally we define the following relation:

$$
M \leq_{\Phi}^{G \max } N \quad \text { iff } \quad L_{M}^{\Phi} \leq_{l e x} L_{N}^{\Phi}
$$

We denote $d_{G \max }$ the function mapping a pair $(M, \Phi)$ to the list $L_{M}^{\Phi}$, and we call this the distance Gmax between the valuation $M$ and the knowledge multi-set $\Phi$.

\section{Theorem 17}

$\Delta^{G m a x}$ defined by

$$
\Delta_{K}^{G \max }(\Phi)=T h\left(\min \left(\bmod (K), \leq_{\Phi}^{G \max }\right)\right)
$$

is an arbitration merging operator. Moreover, if the pseudo-distance $d$ satisfies the Triangle Inequality, then $\Delta^{\text {Gmax }}$ satisfies (ICit).

\section{Concrete merging operators}

This section is devoted to defining concrete operators using the techniques explained in the previous section. Thus, first, we define some distances from which we define our operators.

Remember that in the finite case the Dalal distance [6] between a valuation and a theory is defined using the Hamming distance between valuations, i.e. the distance between $M$ and $N$, is the number of propositional variables in which they differ. For instance, the Hamming distance between $(1,1,1,0,0)$ and $(1,0,1,1,0)$ is equal to 2 because they differ exactly in the second and in the fourth variables.

In the infinite case (when the number of propositional variables is infinite) we define the generalized Dalal distance below. 
First we adopt the following notation: given a valuation $M$, we will write $M(i)$ instead of $M\left(p_{i}\right)$ the value of $M$ in the variable $p_{i}$.

Now, the generalized Dalal distance $d_{1}: \mathcal{V} \times \mathcal{V} \rightarrow \overline{\mathbb{R}^{+}}$is defined by putting

$$
d_{1}(M, N)=\sum_{i=1}^{\infty}|M(i)-N(i)|
$$

This function verifies the condition 1 and 2 of pseudo-distance given at the beginning of Section 2 in page 10. Moreover as the range of this function is $\mathbb{N} \cup\{\infty\}$ we have that for any pair of theories $T, K$ the set $\{d(M, P) ; M \models T, P=K\}$ has a minimum, so the condition 3 is verified. Also, it is clear that $d$ satisfies the Triangle Inequality.

Now we define the discrete distance, $d_{2}: \mathcal{V} \times \mathcal{V} \rightarrow \overline{\mathbb{R}^{+}}$by putting

$$
d_{2}(M, N)= \begin{cases}0 & \text { if, } M=N \\ 1 & \text { if, } M \neq N\end{cases}
$$

The verification that $d_{2}$ satisfies the conditions of pseudo-distance given in 10 is straightforward. The condition 3 is due to the fact that the range of $d_{2}$ is the set $\{0,1\}$. Indeed, $d_{2}$ is a distance, i.e. it satisfies the Triangle Inequality.

It is quite interesting to notice that starting from this discrete distance we have

$$
\leq_{\Phi}^{G \max }=\leq_{\Phi}^{\Sigma}
$$

This is because $L_{\mathrm{M}}^{\Phi}$ is a decreasing sequence of $1^{\prime} s$ and $0^{\prime} s$, since $d(M, K)$ is 1 or 0 for any valuation $M$ and any knowledge base $K$; indeed it is equal to 1 if $M=K$ and it is equal to 0 if $M \not \models K$. Thus, it is clear that $L_{\mathrm{M}}^{\Phi}<_{l e x} L_{\mathrm{N}}^{\Phi}$ if and only if the number of $1^{\prime} s$ in $L_{\mathrm{M}}^{\Phi}$ is less than the number of $1^{\prime} s$ in $L_{\mathrm{N}}^{\Phi}$, and this is equivalent to $\sum_{K \in \Phi} d(M, K)<\sum_{K \in \Phi} d(N, K)$.

Putting together this observation with Corollary 14, we have the following result

\section{Corollary 18}

For the discrete distance we have $\Delta^{\Sigma}=\Delta^{\text {Gmax }}$. Thus, there are merging operators which are of arbitration and majority at the same time.

It is still an open problem to know if the unique operator having a majority and an arbitration behavior at the same time is the previous one. There is some work done in this way in [16]. We conjecture that there is only one operator which satisfaying (Arb) and (Maj).

The third distance we consider is the so called Cantor distance, $d_{3}: \mathcal{V} \times \mathcal{V} \rightarrow \overline{\mathbb{R}^{+}}$defined in the following way:

$$
d_{3}(M, N)=\sum_{i=1}^{\infty} \frac{|M(i)-N(i)|}{2^{i}}
$$


Notice that this distance gives a hierarchy over the propositional variables: the first variable is the most important and the importance decreases as the subscript of the variable increases.

First of all, let us remark this well known fact: the set of valuations with the distance $d_{3}$ (see below) is in fact isometric to Cantor's space; this is the reason to call $d_{3}$ Cantor's distance.

The conditions 1 and 2 of pseudo-distance are clearly satisfied. Also, it is straightforward to verify the Triangle Inequality for $d_{3}$. In order to verify the condition 3 , let us notice that $d_{3}$ is a continuous function mapping the product of Cantor space by itself in $[0,1]$ with the topology inherited of $\mathbb{R}$. But the Cantor space is compact because it is an infinite product of the space $\{0,1\}$ with the discrete topology which is compact, and by the Tychonoff theorem the product of compact spaces is compact. Since $\bmod (T)$ is a closed set for any $T, \bmod (T)$ is compact. Therefore $\bmod (K) \times \bmod (T)$ is compact and so, the continuous function $d_{3}$ takes a minimum value in that set, that is to say the condition 3 holds.

\section{Examples and observations}

In order to distinguish the operators after the distance used to build them we make explicit mention of it. Thus $\Delta^{\Sigma\left(d_{i}\right)}, \Delta^{\max \left(d_{j}\right)}$ and $\Delta^{\operatorname{Gmax}\left(d_{k}\right)}$ are the operators $\Sigma$, Max and Gmax built from $d_{i}, d_{j}$ and $d_{k}$ respectively where $i, j, k \in\{1,2,3\}$.

The following observation tells us that if two sources of the knowledge multi-set totally disagree, then the $\Sigma$ operator and the Max operator built from Dalal distance choose exactly the whole knowledge base representing the integrity constraints. More precisely:

\section{Observation 19}

Let $\Phi=\left\{K_{1}, K_{2}, \ldots, K_{n}\right\}$ be a knowledge multi-set such that there are $K_{i}, K_{j}$ with $d_{1}\left(K_{i}, K_{j}\right)=$ $\infty$. Then for any $R$

$$
\Delta_{R}^{\Sigma\left(d_{1}\right)}(\Phi)=R=\Delta_{R}^{\max \left(d_{1}\right)}(\Phi)
$$

To check this observation notice that since $d_{1}$ satisfies the Triangle Inequality, for any valuation $M$ we have $d\left(M, K_{i}\right)=\infty$ or $d\left(M, K_{j}\right)=\infty$. From this, it follows that $d_{\Sigma}(M, \Phi)=\infty$ and $d_{\max }(M, \Phi)=\infty$. From this and the definitions the equalities above follow.

Thus in case that there is a strong disagreement in the sources of information the operators $\Delta^{\Sigma\left(d_{1}\right)}, \Delta^{\max \left(d_{1}\right)}$ ignore these sources of information.

When $d\left(R, K_{i}\right)<\infty$ for any $K_{i}$ in $\Phi$, then the operator Gmax might give a result different of the constraints.

Let us illustrate the behavior of these operators with some examples.

\section{Example 20}


Let $\Phi=\left\{K_{1}, K_{2}\right\}$ be a knowledge multi-set and $R$ a knowledge base defined by as follows

$$
\begin{aligned}
K_{1} & =\operatorname{Th}(\{M: M(2 k+1)=0, \forall k \geq 0\}) \\
K_{2} & =\operatorname{Th}(\{M: M(2 k+1)=1, \forall k \geq 0\}) \\
R & =\operatorname{Th}(\{M: M(1)=M(3)=0, M(5)=M(7)=M(9)=1\})
\end{aligned}
$$

It is clear that $d_{1}\left(K_{1}, K_{2}\right)=\infty$, then, Observation 19, tells us that $\Delta_{R}^{\Sigma\left(d_{1}\right)}(\Phi)=R=\Delta_{R}^{\max \left(d_{1}\right)}(\Phi)$. We can also verify that for any $M \models R$ satisfying $M(2 k+1)=1, \forall k \geq 5$, we have $d_{1}\left(M, K_{2}\right)=2$; so, $L_{M}^{\Phi}=(\infty, 2)$. It is not hard to see that it is the minimum value in the lexicographical order for $L_{M}^{\Phi}$ when $M \models R$. Thus, we can conclude

$$
\Delta_{R}^{\operatorname{Gmax}\left(d_{1}\right)}(\Phi)=\operatorname{Th}(\{M: M \models R, M(2 n+1)=1 \forall n \geq 5\})
$$

For the discrete distance we have that any $M \models R$ verifies $d_{2}\left(M, K_{1}\right)=d_{2}\left(M, K_{2}\right)=1$. Therefore

$$
\Delta_{R}^{\Sigma\left(d_{2}\right)}(\Phi)=\Delta_{R}^{\max \left(d_{2}\right)}(\Phi)=\Delta_{R}^{\max \left(d_{2}\right)}(\Phi)=R
$$

Now we treat the case of the Cantor distance. To find the distance between $M \models R$ and the knowledge bases $K_{1}$ and $K_{2}$ we proceed as follows: first notice that the unique variables of models of $R$ affecting the distance to $K_{1}$ and $K_{2}$ are the odd variables greater than 9 . Now put $\mathrm{A}_{M}=\{n: M(2 n+1)=0, n \geq 5\}$ and $\mathrm{B}_{M}=\{n: M(2 n+1)=1, n \geq 5\}$, then

$$
d\left(M, K_{1}\right)=\frac{1}{2^{5}}+\frac{1}{2^{7}}+\frac{1}{2^{9}}+\sum_{n \in \mathrm{B}_{M}} \frac{1}{2^{2 n+1}} ; \quad d\left(M, K_{2}\right)=\frac{1}{2}+\frac{1}{2^{3}}+\sum_{n \in \mathrm{A}_{M}} \frac{1}{2^{2 n+1}}
$$

But $\mathrm{A}_{M} \cup \mathrm{B}_{M}=\{n: n \geq 5\}$ and $\mathrm{A}_{M} \cap \mathrm{B}_{M}=\varnothing$ so, for any $M \models R$ we have

$$
d\left(M, K_{1}\right)+d\left(M, K_{2}\right)=\frac{1}{2}+\frac{1}{2^{3}}+\frac{1}{2^{5}}+\frac{1}{2^{7}}+\frac{1}{2^{9}}+\sum_{n \geq 5} \frac{1}{2^{2 n+1}}
$$

Therefore $\Delta_{R}^{\Sigma\left(d_{3}\right)}(\Phi)=R$.

If $\mathrm{A}_{M}=\varnothing$ then

$$
d\left(M, K_{1}\right)=\frac{1}{2^{5}}+\frac{1}{2^{7}}+\frac{1}{2^{9}}+\sum_{n \geq 5} \frac{1}{2^{2 n+1}} ; \quad d\left(M, K_{2}\right)=\frac{1}{2}+\frac{1}{2^{3}}
$$

Thus, $d\left(M, K_{2}\right)>d\left(M, K_{1}\right)$. We can observe that if $\mathrm{A}_{M} \neq \varnothing, d\left(M, K_{2}\right)$ increases. This means that the least value in $\max \left\{d\left(M, K_{1}\right), d\left(M, K_{2}\right)\right\}$ is obtained when $\mathrm{A}_{M}=\varnothing$. Therefore $\min \left\{\max \left\{d\left(M, K_{1}\right), d\left(M, K_{2}\right)\right\}: M \in \bmod (R)\right\}=5 / 2^{3}$, the value of $d\left(M, K_{2}\right)$. This holds for $M(2 n+1)=1 \forall n \geq 5$. So, $\left(5 / 2^{3}, 53 / 2^{9}\right)$ is the minimum in the lexicographical order. Therefore

$$
\Delta_{R}^{\max \left(d_{3}\right)}(\Phi)=\Delta_{R}^{\max \left(d_{3}\right)}(\Phi)=T h\{M \models R: M(2 n+1)=1 \forall n \geq 5\}
$$

\section{Example 21}


Let $\Phi=\left\{K_{1}, K_{2}, K_{3}, K_{4}\right\}$ be a knowledge multi-set, where

$$
\begin{aligned}
& K_{1}=\operatorname{Cn}\left(\left\{p_{2}, \neg p_{3}, \neg p_{4}\right\} \cup\left\{p_{i}: i \geq 6\right\}\right) \\
& K_{2}=\operatorname{Cn}\left(\left\{p_{1}, \neg p_{2}, \neg p_{3}\right\} \cup\left\{p_{i}: i \geq 6\right\}\right) \\
& K_{3}=\operatorname{Cn}\left(\left\{\neg p_{1}, p_{3}, p_{4}, p_{5}\right\} \cup\left\{p_{i}: i \geq 6\right\}\right) \\
& K_{4}=\operatorname{Cn}\left(\left\{p_{1}, p_{2}, p_{3}, \neg p_{4}, \neg p_{5}\right\} \cup\left\{p_{i}: i \geq 6\right\}\right)
\end{aligned}
$$

That is to say

$$
\begin{aligned}
& \bmod \left(K_{1}\right)=(*, 1,0,0, *, \overline{1}) \\
& \bmod \left(K_{2}\right)=(1,0,0, *, *, \overline{1}) \\
& \bmod \left(K_{3}\right)=(0, *, 1,1,1, \overline{1}) \\
& \bmod \left(K_{4}\right)=(1,1,1,0,0, \overline{1})
\end{aligned}
$$

where $\overline{1}$ denotes the sequence equal to 1 and $*$ denotes any value in $\{0,1\}$ and by abuse we identify $(*, 1,0,0, *, \overline{1})$ with the set of models of this form, etc.

Let $R=\operatorname{Cn}\left(\left\{p_{3} \wedge p_{4} \wedge \neg p_{5}\right\}\right)$ so $\bmod (R)=(*, *, 1,1,0, \bar{*})$ We proceed to find the minimum values for $d_{\Sigma}(M, \Phi), d_{\max }(M, \Phi)$ and $d_{G \max }(M, \Phi)$ when $M \models R$ and $d$ is one of $d_{i}$ for $i=1,2,3$. First notice that any model $N$ of $K_{i}$ verifies $N(i)=1$ for all $i \geq 6$. So, the models $M$ of $R$ realizing the minimum values have to verify $M(i)=1$ for all $i \geq 6$. There are four possible cases:

Case $1 M(1)=M(2)=1$, i.e. $M=(1,1,1,1,0, \overline{1})$.

Case $2 M(1)=0, M(2)=1$, i.e. $M=(0,1,1,1,0, \overline{1})$.

Case $3 M(1)=M(2)=0$, i.e. $M=(0,0,1,1,0, \overline{1})$.

Case $4 M(1)=1, M(2)=0$, i.e. $M=(1,0,1,1,0, \overline{1})$.

The Table 1 is very useful to calculate the Dalal distance. In the boxes of the table we find a 1 in the positions in which the models realizing the minimun differ.

In Case 1 we have that the minimum values are

$$
d_{1}\left(M, K_{1}\right)=2 \quad d_{1}\left(M, K_{2}\right)=2 \quad d_{1}\left(M, K_{3}\right)=2 \quad d_{1}\left(M, K_{4}\right)=2
$$

therefore $d_{\Sigma}(M, \Phi)=8, d_{\max }(M, \Phi)=2$ and $d_{G \max }(M, \Phi)=(2,2,2,2)$.

In Case 2 we have that the minimum values are

$$
d_{1}\left(M, K_{1}\right)=2 \quad d_{1}\left(M, K_{2}\right)=3 \quad d_{1}\left(M, K_{3}\right)=0 \quad d_{1}\left(M, K_{4}\right)=2
$$

therefore $d_{\Sigma}(M, \Phi)=7, d_{\max }(M, \Phi)=3$ and $d_{G \max }(M, \Phi)=(3,2,2,0)$. 


\begin{tabular}{|l|l|l|l|l|}
$\left.\underset{\bmod \left(K_{i}\right)}{\bmod (R}\right)$ & $(1,1,1,1,0, \overline{1})$ & $(0,1,1,1,0, \overline{1})$ & $(0,0,1,1,0, \overline{1})$ & $(1,0,1,1,0, \overline{1})$ \\
\hline$(*, 1,0,0, *, \overline{1})$ & $0,0,1,1,0, \overline{0}$ & $0,0,1,1,0, \overline{0}$ & $0,1,1,1,0, \overline{0}$ & $0,1,1,1,0, \overline{0}$ \\
\hline$(1,0,0, *, *, \overline{1})$ & $0,1,1,0,0, \overline{0}$ & $1,1,1,0,0, \overline{0}$ & $1,0,1,0,0, \overline{0}$ & $0,0,1,0,0, \overline{0}$ \\
\hline$(0, *, 1,1,1, \overline{1})$ & $1,0,0,0,1, \overline{0}$ & $0,0,0,0,0, \overline{0}$ & $0,0,0,0,1, \overline{0}$ & $1,0,0,0,1, \overline{0}$ \\
\hline$(1, *, 1,0,0, \overline{1})$ & $0,1,0,1,0, \overline{0}$ & $1,0,0,1,0, \overline{0}$ & $1,0,0,1,0, \overline{0}$ & $0,0,0,1,0, \overline{0}$ \\
\hline
\end{tabular}

Table 1: Calculating Dalal distances.

In Case 3 we have that the minimum values are

$$
d_{1}\left(M, K_{1}\right)=3 \quad d_{1}\left(M, K_{2}\right)=2 \quad d_{1}\left(M, K_{3}\right)=1 \quad d_{1}\left(M, K_{4}\right)=2
$$

therefore $d_{\Sigma}(M, \Phi)=8, d_{\max }(M, \Phi)=3$ and $d_{G \max }(M, \Phi)=(3,2,2,1)$.

In Case 4 we have that the minimum values are

$$
d_{1}\left(M, K_{1}\right)=3 \quad d_{1}\left(M, K_{2}\right)=1 \quad d_{1}\left(M, K_{3}\right)=2 \quad d_{1}\left(M, K_{4}\right)=1
$$

therefore $d_{\Sigma}(M, \Phi)=7, d_{\max }(M, \Phi)=3$ and $d_{G \max }(M, \Phi)=(3,2,1,1)$.

From these observations it follows that

$$
\begin{aligned}
& \bmod \left(\Delta_{R}^{\Sigma\left(d_{1}\right)}(\Phi)\right)=\{(0,1,1,1,0, \overline{1}),(1,0,1,1,0, \overline{1})\} \\
& \bmod \left(\Delta_{R}^{\max \left(d_{1}\right)}(\Phi)\right)=\bmod \left(\Delta_{R}^{\operatorname{Gmax}\left(d_{1}\right)}(\Phi)\right)=\{(1,1,1,1,0, \overline{1})\}
\end{aligned}
$$

Now we treat the discrete distance. Notice that the model $M^{\prime}$ of $R$ defined by $M^{\prime}=(0,1,1,1,0,1,1,1$ is a model of $K_{3}$, but there are no models of $R$ which are models of $K_{1}, K_{2}$ or $K_{4}$.

Thus, for any model $M$ of $R$ different to $M^{\prime}$ we have

$$
d\left(M, K_{1}\right)=d\left(M, K_{2}\right)=d\left(M, K_{3}\right)=d\left(M, K_{4}\right)=1
$$

Moreover

$$
d\left(M^{\prime}, K_{1}\right)=d\left(M^{\prime}, K_{2}\right)=d\left(M^{\prime}, K_{4}\right)=1 \quad d\left(M^{\prime}, K_{3}\right)=0
$$

From these observations we obtain

$$
\Delta_{R}^{\max \left(d_{2}\right)}(\Phi)=R, \quad \bmod \left(\Delta_{R}^{\Sigma\left(d_{2}\right)}(\Phi)\right)=\bmod \left(\Delta_{R}^{G \max \left(d_{2}\right)}(\Phi)\right)=\left\{M^{\prime}\right\}
$$

Finally we deal with Cantor distance. Using the previous table we proceed to find the minimum values. 
Case $1 M(2)=M(3)=1$, i.e. $M=(1,1,1,1,0, \overline{1})$. In this case

$$
\begin{array}{ll}
d_{3}\left(M, K_{1}\right)=\frac{1}{2^{3}}+\frac{1}{2^{4}} & d_{3}\left(M, K_{2}\right)=\frac{1}{2^{2}}+\frac{1}{2^{3}} \\
d_{3}\left(M, K_{3}\right)=\frac{1}{2}+\frac{1}{2^{5}} & d_{3}\left(M, K_{4}\right)=\frac{1}{2^{2}}+\frac{1}{2^{4}}
\end{array}
$$

Therefore

$$
d_{\Sigma}(M, \Phi)=\frac{45}{2^{5}}, d_{\max }(M, \Phi)=\frac{17}{2^{5}}, d_{G \max }(M, \Phi)=\left(\frac{17}{2^{5}}, \frac{3}{2^{3}}, \frac{5}{2^{4}}, \frac{3}{2^{4}}\right)
$$

Case $2 M(2)=1, M(3)=0$, i.e. $M=(0,1,1,1,0, \overline{1})$. In this case

$$
\begin{array}{ll}
d_{3}\left(M, K_{1}\right)=\frac{1}{2^{3}}+\frac{1}{2^{4}} & d_{3}\left(M, K_{2}\right)=\frac{1}{2}+\frac{1}{2^{2}}+\frac{1}{2^{3}} \\
d_{3}\left(M, K_{3}\right)=0 & d_{3}\left(M, K_{4}\right)=\frac{1}{2}+\frac{1}{2^{4}}
\end{array}
$$

Therefore

$$
d_{\Sigma}(M, \Phi)=\frac{13}{2^{3}}, d_{\max }(M, \Phi)=\frac{7}{2^{3}}, d_{G \max }(M, \Phi)=\left(\frac{7}{2^{3}}, \frac{9}{2^{4}}, \frac{3}{2^{4}}, 0\right)
$$

Case $3 M(2)=M(3)=0$, i.e. $M=(0,0,1,1,0, \overline{1})$. In this case

$$
\begin{aligned}
d_{3}\left(M, K_{1}\right) & =\frac{1}{2^{2}}+\frac{1}{2^{3}}+\frac{1}{2^{4}} & d_{3}\left(M, K_{2}\right) & =\frac{1}{2}+\frac{1}{2^{3}} \\
d_{3}\left(M, K_{3}\right) & =\frac{1}{2^{5}} & d_{3}\left(M, K_{4}\right) & =\frac{1}{2}+\frac{1}{2^{4}}
\end{aligned}
$$

Therefore

$$
d_{\Sigma}(M, \Phi)=\frac{53}{2^{5}}, d_{\max }(M, \Phi)=\frac{5}{2^{3}}, d_{\max }(M, \Phi)=\left(\frac{5}{2^{3}}, \frac{9}{2^{4}}, \frac{7}{2^{4}}, \frac{1}{2^{5}}\right)
$$

Case $4 M(2)=0, M(3)=1$, i.e. $M=(1,0,1,1,0, \overline{1})$. In this case

$$
\begin{aligned}
d_{3}\left(M, K_{1}\right) & =\frac{1}{2^{2}}+\frac{1}{2^{3}}+\frac{1}{2^{4}} & d_{3}\left(M, K_{2}\right) & =\frac{1}{2^{3}} \\
d_{3}\left(M, K_{3}\right) & =\frac{1}{2}+\frac{1}{2^{5}} & d_{3}\left(M, K_{4}\right) & =\frac{1}{2^{4}}
\end{aligned}
$$

Therefore

$$
d_{\Sigma}(M, \Phi)=\frac{37}{2^{5}}, d_{\max }(M, \Phi)=\frac{17}{2^{5}}, d_{G \max }(M, \Phi)=\left(\frac{17}{2^{5}}, \frac{7}{2^{4}}, \frac{1}{2^{3}}, \frac{1}{2^{4}}\right)
$$

Finally from the previous observations we obtain:

$$
\begin{aligned}
& \bmod \left(\Delta_{R}^{\Sigma\left(d_{3}\right)}(\Phi)\right)=\{(1,0,1,1,0, \overline{1})\} \\
& \bmod \left(\Delta_{R}^{\max \left(d_{3}\right)}(\Phi)\right)=\{(1,1,1,1,0, \overline{1}),(1,0,1,1,0, \overline{1})\} \\
& \bmod \left(\Delta_{R}^{\max \left(d_{3}\right)}(\Phi)\right)=\{(1,1,1,1,0, \overline{1})\}
\end{aligned}
$$




\section{Final remarks and questions}

There are two dimensions in which the infinite can be introduced in the framework of logic based merging. The language and the number of sources. In this work we have introduced the infinite in the first of these dimensions. That is, we have considered the case of propositional languages with countably many propositional variables. This is important because in many situations we do not know in advance how many variables will be involved. So, we need to have mechanisms to treat these situations. In this setting, the information of one source is a logical theory. In this sense we have joined the classical AGM tradition in which an epistemic state is a logical theory. Then, we have done the lifting of the representation results found in [15] to the case of infinite propositional logic.

The introduction of the infinite in the second dimension, that is, to consider the possibility of having an infinite number of sources, is an issue not addressed in this work. Although we think this is an important theoretical issue and that the basic postulates have a generalization quite straightforward, we do not have a clear idea about what a majority behavior is in this case. Serious problems arise, inherent to the infinite, when we try to determine the majority; see for instance the recent work of Pacuit and Salame [20]. In that work, either the set of even numbers or the set of odd numbers is a majority set in the set of natural numbers. That is quite surprising. On the other hand, the naive notion of majority, based on cardinality, does not work well either.

A by-product of studying the infinite framework for merging operators is the possibility of finding the correct and more general formulation for some postulates (e.g. the majority postulate). The infinite case tells us that the distances used to define operators have to be very particular: they have to satisfy the condition 3 in Definition 12, i.e. a realization condition which is satisfied, for instance, by continuous functions over compact spaces. Another very interesting fact is the necessity of considering the smoothness property which guarantees the consistency of operators defined by smooth assignments. This kind of property has been introduced by Kraus et al. in [17] in order to have representation theorems for Nonmonotonic relations.

A challenging question is to study the behaviour of operators when we restrain the co-domain and the nature of information. For instance, what happens if instead of $\mathcal{T}$ (the set of all theories) we take the set of theories finitely generated? Do the representation theorems hold? What is not very clear for us is the nature of the assignments which could guarantee that the set of the minimal models of a finitely generated theory be indeed a set of models whose theory is finitely generated.

A natural generalization of this work is to consider, instead of theories, more general epistemic states as the encoding of the information given by a source.

To finish these concluding remarks we would like to say that the treatment of the infinite done in this work can be viewed as the beginning of a possible extension of merging to the framework of First Order Logic. 


\section{Acknowledgements}

We would like to thank the anonymous referees for their observations which have contributed to improve the quality of this work. The second author thanks the Consejo de Desarrollo Científico, Humanístico y Tecnológico (CDCHT) from the Universidad de Los Andes for the partial financial support for this work.

\section{A Proofs}

In order to prove Theorem 4 we state and show some propositions and lemmas. Next proposition is a useful standard tool in many of the proofs below. It is so standard, that most of the time we do not even mention it. It is stated without proof.

\section{Proposition 22}

Let $K$ and $R$ theories. Then

(i) $\bmod (K \cap R)=\bmod (K) \cup \bmod (R)$

(ii) $\bmod (\mathrm{Cn}(K \cup R))=\bmod (K) \cap \bmod (R)$

(iii) $K \subseteq R \quad \Longleftrightarrow \quad \bmod (K) \supseteq \bmod (R)$

\section{Lemma 23}

For any valuation $M$ and any knowledge multi-set $\Phi$, there exists $K$ such that $M \models \Delta_{K}(\Phi)$.

Proof: Take $K=T h(\{M\})$. Clearly $M$ is the unique model of $K$. Now use (IC0) to conclude.

\section{Lemma 24}

Assume that $\Delta$ satisfies (ICO), (IC6) and (ICr). Then, if $\Delta_{K \cap T}(\Phi) \cup K \vdash \perp$ necessarily $\Delta_{K \cap T}(\Phi)=\Delta_{T}(\Phi)$.

Proof: We have by $(\mathrm{IC} 0)$ that $\Delta_{K \cap T}(\Phi) \supseteq K \cap T$, i.e. $\bmod \left(\Delta_{K \cap T}(\Phi)\right) \subseteq \bmod (K \cap T)=$ $\bmod (K) \cup \bmod (T)$. By the assumption $\bmod \left(\Delta_{K \cap T}(\Phi)\right) \cap \bmod (K)=\varnothing$. From these two things, it follows $\bmod \left(\Delta_{K \cap T}(\Phi)\right) \subseteq \bmod (T)$, i.e. $\Delta_{K \cap T}(\Phi) \supseteq T$. In particular $\Delta_{K \cap T}(\Phi) \cup T=$ $\Delta_{K \cap T}(\Phi)$ (because, as is well known $\bmod \left(\Delta_{K \cap T}(\Phi) \cup T\right)=\bmod \left(\Delta_{K \cap T}(\Phi)\right) \cap \bmod (T)$ and by the previous observation the last expression is $\bmod \left(\Delta_{K \cap T}(\Phi)\right)$ which is non empty for IC0). Therefore $\Delta_{K \cap T}(\Phi) \cup T$ is consistent. From this, by (IC6), ( IC7) we have $\operatorname{Cn}\left(\Delta_{K \cap T}(\Phi) \cup T\right)=$ $\Delta_{\mathrm{Cn}((K \cap T) \cup T)}(\Phi)$ that is to say $\Delta_{K \cap T}(\Phi)=\Delta_{T}(\Phi)$.

\section{Proposition 25}

Assume that $\Delta$ satisfies (ICO), (IC6) and (ICr). Then for any valuation $M$

$$
M \models \Delta_{K}(\Phi) \wedge M \models \Delta_{T}(\Phi) \Rightarrow M \models \Delta_{K \cap T}(\Phi)
$$

Proof: We consider two cases. First, we suppose that $\Delta_{K \cap T}(\Phi) \cup K$ is not consistent. By the lemma $24 \Delta_{K \cap T}(\Phi)=\Delta_{T}(\Phi)$. From what follows $M \models \Delta_{K \cap T}(\Phi)$.

In the second case, i.e. $\Delta_{K \cap T}(\Phi) \cup K$ is consistent, by (IC6), (IC7) we have $\operatorname{Cn}\left(\Delta_{K \cap T}(\Phi) \cup\right.$ $K)=\Delta_{\operatorname{Cn}((K \cap T) \cup K)}(\Phi)$. Therefore $\operatorname{Cn}\left(\Delta_{K \cap T}(\Phi) \cup K\right)=\Delta_{K}(\Phi)$. Thus, $M \models \Delta_{K \cap T}(\Phi) \cup$ 
$K$. In particular $M \models \Delta_{K \cap T}(\Phi)$.

Next proposition is the trichotomy property for merging operators

\section{Proposition 26}

Assume that $\Delta$ satisfies (IC0), (IC6) and (IC7). Then

$$
\Delta_{K \cap T}(\Phi)= \begin{cases}\Delta_{K}(\Phi), & \text { if } \Delta_{K \cap T}(\Phi) \cup T \vdash \perp \\ \Delta_{T}(\Phi), & \text { if } \Delta_{K \cap T}(\Phi) \cup K \vdash \perp \\ \Delta_{K}(\Phi) \cap \Delta_{T}(\Phi), & \text { otherwise }\end{cases}
$$

Proof: The two first cases follow from lemma 24. Thus, let us suppose that $\Delta_{K \cap T}(\Phi) \cup K \nvdash$ $\perp, \Delta_{K \cap T}(\Phi) \cup T \nvdash \perp$. By (IC6), (IC7) $\mathrm{C}_{\mathrm{n}}\left(\Delta_{K \cap T}(\Phi) \cup K\right)=\Delta_{K}(\Phi)$ and $\mathrm{Cn}_{\mathrm{n}}\left(\Delta_{K \cap T}(\Phi) \cup T\right)=$ $\Delta_{T}(\Phi)$. Therefore

$$
\bmod \left(\Delta_{K \cap T}(\Phi) \cup T\right) \cup \bmod \left(\Delta_{K \cap T}(\Phi) \cup K\right)=\bmod \left(\Delta_{T}(\Phi)\right) \cup \bmod \left(\Delta_{K}(\Phi)\right)
$$

Now, using the distributivity of $\cap$ and $\cup$, it is easy to see that

$$
\left.\bmod \left(\Delta_{K \cap T}(\Phi) \cup T\right) \cup \bmod \left(\Delta_{K \cap T}(\Phi) \cup K\right)\right)=\bmod \left(\Delta_{K \cap T}(\Phi)\right) \cap \bmod (K \cap T)
$$

By $(\mathrm{IC0}) \bmod \left(\Delta_{K \cap T}(\Phi)\right) \subseteq(\bmod (K \cap T))$, so the right hand side of 4 is $\bmod \left(\Delta_{K \cap T}(\Phi)\right)$. Using equations 3 and 4 and transitivity we get, $\bmod \left(\Delta_{K \cap R}(\Phi)\right)=\bmod \left(\Delta_{K}(\Phi) \cap \Delta_{R}(\Phi)\right)$, i.e. $\Delta_{K \cap R}(\Phi)=\Delta_{K}(\Phi) \cap \Delta_{R}(\Phi)$.

\section{Lemma 27}

If $\Delta$ satisfies (IC6) then for any valuation $M$,

$$
M \models \Delta_{K}(\Phi) \wedge M \models T \Rightarrow M \models \Delta_{\mathrm{Cn}(K \cup T)}(\Phi)
$$

Proof: By (IC6) we have $\operatorname{Cn}\left(\Delta_{K}(\Phi) \cup T\right) \supseteq \Delta_{\mathrm{Cn}(K \cup T)}(\Phi)$, i.e. $\bmod \left(\Delta_{K}(\Phi) \cup T\right) \subseteq \bmod \left(\Delta_{\mathrm{Cn}(K \cup T)}(\right.$ As $M \models \Delta_{K}(\Phi) \cup T$ necessarily $M \models \Delta_{\mathrm{Cn}(K \cup T)}(\Phi)$.

\section{Lemma 28}

Assume that $\Delta$ satisfies (IC0), (IC6) and (IC7). Then for any valuations $M$ and $N$,

$$
M \models \Delta_{K}(\Phi) \wedge N \models \Delta_{T}(\Phi) \Rightarrow M \models \Delta_{K \cap T}(\Phi) \vee N \models \Delta_{K \cap T}(\Phi)
$$

Proof: This follows of Proposition 26. Indeed, we consider the three possible cases for $\Delta_{K \cap T}(\Phi)$. If $\Delta_{K \cap T}(\Phi)=\Delta_{K}(\Phi)$, then $M \models \Delta_{K \cap T}(\Phi)$. If $\Delta_{K \cap T}(\Phi)=\Delta_{T}(\Phi)$, then $N \models \Delta_{K \cap T}(\Phi)$. Finally, if $\Delta_{K \cap T}(\Phi)=\Delta_{K}(\Phi) \cap \Delta_{T}(\Phi)$, then $\Delta_{K \cap T}(\Phi) \subseteq \Delta_{K}(\Phi)$ and $\Delta_{K \cap T}(\Phi) \subseteq \Delta_{T}(\Phi)$. So, $\bmod \left(\Delta_{K \cap T}(\Phi)\right) \supseteq \bmod \left(\Delta_{K}(\Phi)\right)$ and $\bmod \left(\Delta_{K \cap T}(\Phi)\right) \supseteq \bmod \left(\Delta_{T}(\Phi)\right)$. Therefore $M, N \models$ $\Delta_{K \cap T}(\Phi)$.

\section{Lemma 29}

Assume that $\Delta$ satisfies (IC7). Then if $\Delta_{K}(\Phi) \cup T$ is consistent, then $\Delta_{\operatorname{Cn}(K \cup T)}(\Phi) \supseteq \Delta_{K}(\Phi)$. 
Proof: By (IC7) we have that $\Delta_{\mathrm{Cn}(K \cup T)}(\Phi) \supseteq \mathrm{Cn}_{\mathrm{n}}\left(\Delta_{K}(\Phi) \cup T\right)$. Therefore $\Delta_{\mathrm{Cn}(K \cup T)}(\Phi) \supseteq$ $\Delta_{K}(\Phi)$.

The following proposition is the key result for studying the properties of the relation $\leq_{\Phi}$.

\section{Proposition 30}

Assume that $\Delta$ satisfies (IC0), (IC6) and (IC7). Then

$$
M \leq_{\Phi} N \Leftrightarrow \exists K, \exists T\left(M \models \Delta_{K}(\Phi), N \models \Delta_{T}(\Phi), M \models \Delta_{K \cap T}(\Phi)\right)
$$

Proof: The implication $(\Rightarrow)$ is straightforward by the Definition 5 . For the converse, assume that $M \models \Delta_{K}(\Phi), N \models \Delta_{T}(\Phi), M \models \Delta_{K \cap T}(\Phi)$ for one $K$ and one $T$. Let $K^{\prime}$ and $T^{\prime}$ theories such that $M \models \Delta_{K^{\prime}}(\Phi)$ and $N \models \Delta_{T^{\prime}}(\Phi)$. We want to show that $M \models \Delta_{K^{\prime} \cap T^{\prime}}(\Phi)$. By Lemma 28, $M \models \Delta_{K^{\prime} \cap T^{\prime}}(\Phi)$ or $N \models \Delta_{K^{\prime} \cap T^{\prime}}(\Phi)$. By the hypothesis $M$ and $N$ are both models of $K \cap T$. Therefore, $M$ or $N$ is a model of $\Delta_{K^{\prime} \cap T^{\prime}}(\Phi) \cup(K \cap T)$ and $M$ or $N$ is a model of $\Delta_{K \cap T}(\Phi) \cup\left(K^{\prime} \cap T^{\prime}\right)$. That is, both previous sets are consistent. Thus, by (IC6)-(IC7) we get:

$$
\begin{aligned}
& \operatorname{Cn}\left(\Delta_{K^{\prime} \cap T^{\prime}}(\Phi) \cup(K \cap T)\right)=\Delta_{\mathrm{Cn}\left(\left(K^{\prime} \cap T^{\prime}\right) \cup(K \cap T)\right)}(\Phi) \\
& \operatorname{Cn}\left(\Delta_{K \cap T}(\Phi) \cup\left(K^{\prime} \cap T^{\prime}\right)\right)=\Delta_{\mathrm{Cn}\left((K \cap T) \cup\left(K^{\prime} \cap T^{\prime}\right)\right)}(\Phi)
\end{aligned}
$$

From these two equations, by transitivity, we get $\operatorname{Cn}\left(\Delta_{K^{\prime} \cap T^{\prime}}(\Phi) \cup(K \cap T)\right)=\operatorname{Cn}\left(\Delta_{K \cap T}(\Phi) \cup\right.$ $\left.\left(K^{\prime} \cap T^{\prime}\right)\right)$. But $M \models \Delta_{K \cap T}(\Phi) \cup\left(K^{\prime} \cap T^{\prime}\right)$; so, $M \models \Delta_{K^{\prime} \cap T^{\prime}}(\Phi) \cup(K \cap T)$. In particular $M \models \Delta_{K^{\prime} \cap T^{\prime}}(\Phi)$.

In order to see that the relation $\leq_{\Phi}$ of Definition 5 is a total pre-order, it is useful to consider the strict relation $<_{\Phi}$ and the indifference relation $\simeq_{\Phi}$ defined in the following way $M<_{\Phi} N$ iff $M \leq_{\Phi} N$ and $N \not_{\Phi} M$, and $M \simeq_{\Phi} N$ iff $M \leq_{\Phi} N$ and $N \leq_{\Phi} M$.

The following lemma is straightforward consequence of the previous proposition and of the definition of $<_{\Phi}$

\section{Lemma 31}

Assume that $\Delta$ satisfies (IC0), (IC6) and (IC7). Then

$$
M<_{\Phi} N \Leftrightarrow \exists K, \exists T\left(M \models \Delta_{K}(\Phi), N \models \Delta_{T}(\Phi), M \models \Delta_{K \cap T}(\Phi), N \not \models \Delta_{K \cap T}(\Phi)\right)
$$

The following result is an important characterization of $<_{\Phi}$

\section{Lemma 32}

Assume that $\Delta$ satisfies (IC0), (IC6) and (IC7). Then

$$
M<_{\Phi} N \Leftrightarrow \forall K\left(N \models \Delta_{K}(\Phi) \Rightarrow M \not \models K\right)
$$


Proof: $(\Rightarrow)$ Suppose that $M<_{\Phi} N$. Towards a contradiction, let $K$ be a theory such that $N \models$ $\Delta_{K}(\Phi)$ and $M \models K$. Let $T$ such that $M \models \Delta_{T}(\Phi)$ (by virtue of Lemma 23 such a $T$ exists). By Lemma $27, M \models \Delta_{\mathrm{Cn}(K \cup T)}(\Phi)$. As $M<_{\Phi} N$, by Proposition 30, $N \not \Delta_{\mathrm{Cn}(K \cup T) \cap K}(\Phi)$. But $\operatorname{Cn}(K \cup T) \cap K=K$ so, $N \not \models \Delta_{K}(\Phi)$ which is a contradiction.

$(\Leftarrow)$ Assume that for any $R$, if $N \models \Delta_{R}(\Phi)$ then $M \not \models R$. Let $K$ and $T$ be theories such that $M \models \Delta_{K}(\Phi)$ and $N \models \Delta_{T}(\Phi)$. We want to show that $M \models \Delta_{K \cap T}(\Phi)$ and $N \forall \Delta_{K \cap T}(\Phi)$. By Lemma $28, M \models \Delta_{K \cap T}(\Phi)$ or $N \models \Delta_{K \cap T}(\Phi)$. Let us see that $N \models \Delta_{K \cap T}(\Phi)$ does not hold. Otherwise, by hypothesis $M \not \models K \cap T$, but $M \models \Delta_{K}(\Phi)$. Thus, by (IC0), $M \models K$ a contradiction. Then, necessarily, $M \| \Delta_{K \cap T}(\Phi)$.

Now we are going to begin the proof of the fact that $\leq_{\Phi}$ is a total preorder. Note that the previous lemmas use at most the postulates (IC0), (IC6) and (IC7).

\section{Lemma 33}

Assume that $\Delta$ satisfies (IC0), (IC6) and (IC7). Then $<_{\Phi}$ is transitive.

Proof: Suppose that $M<_{\Phi} N$ and $N<_{\Phi} P$. We want to show that $M<_{\Phi} P$. Towards a contradiction, suppose that $M \nless_{\Phi} P$. By Lemma 32, there is $K$ such that $P \models \Delta_{K}(\Phi)$ and $M \models K$. As $N<_{\Phi} P$, for any $T$ verifying $N \models \Delta_{T}(\Phi)$, necessarily $N \models \Delta_{K \cap T}(\Phi)$; as $M<{ }_{\Phi} N$, by Lemma $32, M \forall \forall K \cap T$, which contradicts $M \models K$.

Next lemma says us that the relation $<_{\Phi}$ satisfies one of the properties of modular relations.

\section{Lemma 34}

Assume that $\Delta$ satisfies (ICO), (IC6) and (IC7). Then

$$
\left(M \nless_{\Phi} N \wedge N \nless_{\Phi} M \wedge M<_{\Phi} P\right) \Rightarrow N<_{\Phi} P
$$

Proof: Let $K, T, R$ be theories such that $M \models \Delta_{K}(\Phi), N \models \Delta_{T}(\Phi)$, and $P \models \Delta_{R}(\Phi)$ (the existence of such theories is guaranteed by Lemma 23). By Lemmas 28 and 31, $M, N \models$ $\Delta_{K \cap T}(\Phi)$. As $M<_{\Phi} P$, by Proposition 30, $P \forall \Delta_{K \cap T \cap R}(\Phi)$. By Lemma 28, $N \models$ $\Delta_{K \cap T \cap R}(\Phi)$. By Lemma $31, N<_{\Phi} P$.

The Lemmas 23 and 32 have as a straightforward consequence that $<_{\Phi}$ is irreflexive. This is stated in the following lemma:

\section{Lemma 35}

Assume that $\Delta$ satisfies (ICO), (IC6) and (IC7). Then for any valuation $M, M \nless_{\Phi} M$.

Lemmas 33, 34 and 35 ensure that $<_{\Phi}$ is a modular relation (actually it is a well-known fact that if a relation $<$ is irreflexive and transitive the conclusion in lemma 34 is equivalent to say that the relation is modular). Also it is a well-known fact that in this case, the relation $\preceq$ associated to $<_{\Phi}$ by putting $M \preceq N$ iff $M<_{\Phi} N$ or $M \nless_{\Phi} N$ and $N \nless_{\Phi} M$, is exactly the relation $\leq_{\Phi}$ and moreover it is a total pre-order. Thus, using these observations, we get the following result: 


\section{Proposition 36}

Assume that $\Delta$ satisfies (IC0), (IC6) and (IC7). Then $\leq_{\Phi}$ is a total pre-order.

The following two lemmas are useful proving that an arbitration merging operator induces a majority syncretic assignment which represents it in the sense of equation 1.

\section{Lemma 37}

Assume that $\Delta$ satisfies (IC0), (IC4), (IC5), (IC6) and (IC7). If $\Delta_{R_{1}}\left(\Phi_{1}\right) \cup \Delta_{R_{2}}\left(\Phi_{2}\right) \forall \perp$, then $\Delta_{\mathrm{Cn}\left(R_{1} \cup R_{2}\right)}\left(\Phi_{1} \sqcup \Phi_{2}\right)=\operatorname{Cn}\left(\Delta_{R_{1}}\left(\Phi_{1}\right) \cup \Delta_{R_{2}}\left(\Phi_{2}\right)\right)$.

Proof: By hypothesis, there exists $M \models \Delta_{R_{1}}\left(\Phi_{1}\right) \cup \Delta_{R_{2}}\left(\Phi_{2}\right)$. By (IC0), $M \models R_{1}, R_{2}$, and therefore $M=\Delta_{R_{1}}\left(\Phi_{1}\right) \cup R_{2}$, and $M \models \Delta_{R_{2}}\left(\Phi_{2}\right) \cup R_{1}$. By (IC6) and (IC7),

$$
\begin{aligned}
& \operatorname{Cn}\left(\Delta_{R_{1}}\left(\Phi_{1}\right) \cup R_{2}\right)=\Delta_{\mathrm{Cn}\left(R_{1} \cup R_{2}\right)}\left(\Phi_{1}\right) \\
& \operatorname{Cn}\left(\Delta_{R_{2}}\left(\Phi_{2}\right) \cup R_{1}\right)=\Delta_{\operatorname{Cn}\left(R_{1} \cup R_{2}\right)}\left(\Phi_{2}\right)
\end{aligned}
$$

Therefore $M \models \Delta_{\mathrm{Cn}\left(R_{1} \cup R_{2}\right)}\left(\Phi_{1}\right) \cup \Delta_{\mathrm{Cn}\left(R_{1} \cup R_{2}\right)}\left(\Phi_{2}\right)$. By (IC4) and (IC5),

$$
\mathrm{Cn}\left(\Delta_{\mathrm{Cn}\left(R_{1} \cup R_{2}\right)}\left(\Phi_{1}\right) \cup \Delta_{\mathrm{Cn}\left(R_{1} \cup R_{2}\right)}\left(\Phi_{2}\right)\right)=\Delta_{\mathrm{Cn}\left(R_{1} \cup R_{2}\right)}\left(\Phi_{1} \sqcup \Phi_{2}\right)
$$

Since $\operatorname{Cn}\left(\Delta_{\mathrm{Cn}\left(R_{1} \cup R_{2}\right)}\left(\Phi_{1}\right) \cup \Delta_{\mathrm{Cn}\left(R_{1} \cup R_{2}\right.}\left(\Phi_{2}\right)\right)=\operatorname{Cn}\left(\operatorname{Cn}\left(\Delta_{R_{1}}\left(\Phi_{1}\right) \cup R_{2}\right) \cup \operatorname{Cn}\left(\Delta_{R_{2}}\left(\Phi_{2}\right) \cup R_{1}\right)\right)$ we get easily $\operatorname{Cn}\left(\Delta_{\operatorname{Cn}\left(R_{1} \cup R_{2}\right)}\left(\Phi_{1}\right) \cup \Delta_{\mathrm{Cn}\left(R_{1} \cup R_{2}\right.}\left(\Phi_{2}\right)\right)=\operatorname{Cn}\left(\Delta_{R_{1}}\left(\Phi_{1}\right) \cup R_{2} \cup \Delta_{R_{2}}\left(\Phi_{2}\right) \cup R_{1}\right)$. By (IC0), $\Delta_{R_{1}}\left(\Phi_{1}\right) \supseteq R_{1}$ and $\Delta_{R_{2}}\left(\Phi_{2}\right) \supseteq R_{2}$, so,

$$
\operatorname{Cn}\left(\Delta_{\operatorname{Cn}\left(R_{1} \cup R_{2}\right)}\left(\Phi_{1}\right) \cup \Delta_{\mathrm{Cn}\left(R_{1} \cup R_{2}\right.}\left(\Phi_{2}\right)\right)=\operatorname{Cn}\left(\Delta_{R_{1}}\left(\Phi_{1}\right) \cup \Delta_{R_{2}}\left(\Phi_{2}\right)\right)
$$

From equations 5,6 , and transitivity of the equality, we get finally

$$
\Delta_{\mathrm{Cn}\left(R_{1} \cup R_{2}\right)}\left(\Phi_{1} \sqcup \Phi_{2}\right)=\operatorname{Cn}\left(\Delta_{R_{1}}\left(\Phi_{1}\right) \cup \Delta_{R_{2}}\left(\Phi_{2}\right)\right)
$$

\section{Lemma 38}

Assume that $\Delta$ satisfies (IC0) and (IC1). If $M \neq N$, then there are $R, T \in \mathcal{T}$ such that $M \models$ $\Delta_{R}(\Phi), N \models \Delta_{T}(\Phi)$ and $\Delta_{R}(\Phi) \cup \Delta_{T}(\Phi) \vdash \perp$

Proof: Just take $R=T h(\{M\})$ and $T=T h(\{N\})$.

\section{Lemma 39}

If $M \models \Delta_{K}(\Phi)$, then $M \in \min \left(\bmod (K), \leq_{\Phi}\right)$.

Proof: Suppose $M \models \Delta_{K}(\Phi)$. By (IC0) $M \models K$. Let $N \models K$. By Lemma $32, N \nless_{\Phi} M$. Therefore, by totality of $\leq_{\Phi}, M \leq_{\Phi} N$. This finish the proof of the lemma.

Now we are going to prove that $\Phi \mapsto \leq_{\Phi}$ is a syncretic assignment when $\Delta$ is a merging operator. We have settled that $\leq_{\Phi}$ is a total pre-order. It remains to prove the conditions 1-6 of Definition 3. Actually, we prove a finer result: 


\section{Proposition 40}

Assume that $\Delta$ satisfies (IC0), (IC6) and (IC7). Let $\Phi \mapsto \leq_{\Phi}$ the assignment induced by $\Delta$ using Definition 5. Then the following conditions hold:

(i) If $\Delta$ satisfies (IC2) then $M \models \mathbb{A} \Phi, N \models \mathbb{A} \Phi \Rightarrow M \simeq_{\Phi} N$, i.e. condition 1 of Definition 3 holds. Also condition 2 of Definition 3 holds, i.e. $M \models \mathbb{A} \Phi, N \forall \mathbb{A} \Phi \Rightarrow M<_{\Phi} N$.

(ii) If $\Delta$ satisfies (IC1) and (IC3) then for any consistent theories $K$ and $T \forall M \models K \quad \exists N \models$

$T$ such that $N \leq K \sqcup T M$, i.e. condition 3 of Definition 3.

(iii) If $\Delta$ satisfies (IC4) then for any $M, N, \Phi_{1}, \Phi_{2}, M \leq_{\Phi_{1}} N$ and $M \leq_{\Phi_{2}} N$ entail $M \leq_{\Phi_{1} \sqcup \Phi_{2}}$ $N$, i.e. condition 4 of Definition 3.

(iv) If $\Delta$ satisfies (IC5) then for any $M, N, \Phi_{1}, \Phi_{2}, M<_{\Phi_{1}} N$ and $M \leq_{\Phi_{2}} N$ entail $M<_{\Phi_{1} \sqcup \Phi_{2}}$ $N$, i.e. condition 5 of Definition 3.

(v) If $\Delta$ satisfies (IC5') then for any $M, N, \Phi_{1}, \Phi_{2}, M<_{\Phi_{1}} N$ and $M<_{\Phi_{2}} N$ entail $M<_{\Phi_{1}} \sqcup \Phi_{2}$ $N$, i.e. condition $5^{\prime}$ in the definition of quasi-syncretic assignment.

(vi) $\leq_{\Phi}$ is smooth.

(vii) Furthermore, if $\Delta$ satisfies (Maj) then the assignment satisfies condition 7 of majority syncretic assignment.

Proof: (i) First we prove the condition 1, i.e. $M \simeq_{\Phi} N$ under the hypothesis $M \models \wedge \Phi$ and $N \models$ $\bigwedge \Phi$. Suppose that $M \models \Delta_{K}(\Phi)$ and $N \models \Delta_{T}(\Phi)$. We want to show that $M, N \models \Delta_{K \cap T}(\Phi)$, what, by definition of $\leq_{\Phi}$ (Definition 5), entails $M \simeq_{\Phi} N$. By (IC0) $M \models K, N \models T$, therefore $M, N \models K \cap T$. This, together with the hypothesis, entails $(\mathbb{\wedge} \Phi) \cup(K \cap T)$ is consistent, moreover $M, N \models(\mathbb{A} \Phi) \cup(K \cap T)$. By $(\mathrm{IC} 2) \Delta_{K \cap T}(\Phi)=\operatorname{Cn}((\mathbb{A} \Phi) \cup(K \cap T))$. Therefore $M, N \models \Delta_{K \cap T}(\Phi)$.

Now, we prove the condition 2, i.e. $M \models \mathbb{A} \Phi, N \not \models \mathbb{} \Phi \Rightarrow M<_{\Phi} N$. It is enough to see that $N \forall \Delta_{K \cap T}(\Phi)$ under the assumptions $M \models \Delta_{K}(\Phi)$ and $N \models \Delta_{T}(\Phi)$. Thus, suppose the assumptions hold. If $\Delta_{K \cap T}(\Phi) \cup T$ is consistent, by (IC7), and the fact that $(K \cap T) \cup T=T$, we have $\Delta_{T}(\Phi) \supseteq \Delta_{K \cap T}(\Phi)$. Since $M \models(\mathbb{A} \Phi) \cup(K \cap T)$, by $(\mathrm{IC} 2), \Delta_{K \cap T}(\Phi)=\operatorname{Cn}((\mathbb{\wedge} \Phi) \cup$ $(K \cap T)$ ). This equality says us that $N \not \Delta_{K \cap T}(\Phi)$, otherwise $N \models \mathbb{A} \Phi$, a contradiction.

(ii) Let $K$ and $T$ be two consistent theories. We want to show that for any $M \models K$, there exists $N \models T$, such that $N \leq_{K \sqcup T} M$. We claim that $\Delta_{K \cap T}(K \sqcup T) \cup T \nvdash \perp$. Towards a contradiction suppose $\Delta_{K \cap T}(K \sqcup T) \cup T \vdash \perp$. By (IC0) $\Delta_{K \cap T}(K \sqcup T) \supseteq K \cap T$. From these two facts, it is easy to get using semantical arguments (Proposition 22) that $\Delta_{K \cap T}(K \sqcup T) \supseteq K$, that is $\bmod \left(\Delta_{K \cap T}(K \sqcup T)\right) \subseteq \bmod (K)$. Since $K \cap T$ is consistent, by (IC1) $\Delta_{K \cap T}(K \sqcup T)$ is consistent too. Therefore $\Delta_{K \cap T}(K \sqcup T) \cup K \nvdash \perp$. But $K \supseteq(K \cap T), T \supseteq(K \cap T)$ so, by (IC3) $\Delta_{K \cap T}(K \sqcup T) \cup T \nvdash \perp$, a contradiction. Now, by the claim there exists $N \models T$ and $N \models \Delta_{K \cap T}(K \sqcup T)$. But, if $M \models K, M \models K \cap T$ so, by Lemma $32 M \nless K \sqcup T N$. From this, together with the totality of $\leq_{K \sqcup T}$, follows $N \leq_{K \sqcup T} M$.

(iii) Assume that $M \leq_{\Phi_{1}} N$ and $M \leq_{\Phi_{2}} N$. We want to show that $M \leq_{\Phi_{1} \sqcup \Phi_{2}} N$. Let $K, T, R, S$ be theories such that $M \models \Delta_{K}\left(\Phi_{1}\right), M \models \Delta_{T}\left(\Phi_{2}\right), N \models \Delta_{R}\left(\Phi_{1}\right)$ and $N \models \Delta_{S}\left(\Phi_{2}\right)$ By (IC0), $M \models \Delta_{K}\left(\Phi_{1}\right) \cup T$ and $M \models \Delta_{T}\left(\Phi_{2}\right) \cup K$. From this, using (IC6) and (IC7), follows $\operatorname{Cn}\left(\Delta_{K}\left(\Phi_{1}\right) \cup T\right)=\Delta_{\operatorname{Cn}(K \cup T)}\left(\Phi_{1}\right)$ and $\operatorname{Cn}\left(\Delta_{T}\left(\Phi_{2}\right) \cup K\right)=\Delta_{\operatorname{Cn}(K \cup T)}\left(\Phi_{2}\right)$. Therefore 
$M \models \Delta_{\mathrm{Cn}(K \cup T)}\left(\Phi_{1}\right) \cup \Delta_{\mathrm{Cn}(K \cup T)}\left(\Phi_{2}\right)$. By $(\mathrm{IC} 4)$,

$$
\operatorname{Cn}\left(\Delta_{\mathrm{Cn}(K \cup T)}\left(\Phi_{1}\right) \cup \Delta_{\mathrm{Cn}(K \cup T)}\left(\Phi_{2}\right)\right) \supseteq \Delta_{\mathrm{Cn}(K \cup T)}\left(\Phi_{1} \sqcup \Phi_{2}\right)
$$

So

$$
M \models \Delta_{\mathrm{Cn}(K \cup T)}\left(\Phi_{1} \sqcup \Phi_{2}\right)
$$

With an analogous reasoning, we get

$$
N \models \Delta_{\mathrm{Cn}(R \cup S)}\left(\Phi_{1} \sqcup \Phi_{2}\right)
$$

and $N \models \Delta_{\mathrm{Cn}(R \cup S)}\left(\Phi_{1}\right)$ and $N \models \Delta_{\mathrm{Cn}(R \cup S)}\left(\Phi_{2}\right)$. By hypothesis $M \leq_{\Phi_{1}} N$ and $M \leq_{\Phi_{2}} N$. Then, using Definition 5, we get $M \models \Delta_{\operatorname{Cn}(K \cup T) \cap \operatorname{Cn}(R \cup S)}\left(\Phi_{1}\right)$ and $M \models \Delta_{\operatorname{Cn}(K \cup T) \cap \operatorname{Cn}(R \cup S)}\left(\Phi_{2}\right)$. By (IC4)

$$
\mathrm{Cn}\left(\Delta_{\mathrm{Cn}(K \cup T) \cap \operatorname{Cn}(R \cup S)}\left(\Phi_{1}\right) \cup \Delta_{\mathrm{Cn}(K \cup T) \cap \operatorname{Cn}(R \cup S)}\left(\Phi_{2}\right)\right) \supseteq \Delta_{\mathrm{Cn}(K \cup T) \cap \operatorname{Cn}(R \cup S)}\left(\Phi_{1} \sqcup \Phi_{2}\right)
$$

Therefore $M \models \Delta_{\operatorname{Cn}(K \cup T) \cap \operatorname{Cn}(R \cup S)}\left(\Phi_{1} \sqcup \Phi_{2}\right)$. This, together with the statements 7 and 8 (above) and Proposition 30, entails $M \leq_{\Phi_{1} \sqcup \Phi_{2}} N$.

(iv) Assume that $M<{ }_{\Phi_{1}} N$ and $M \leq_{\Phi_{2}} N$. We want to show that $M<_{\Phi_{1} \sqcup \Phi_{2}} N$. Since $\leq_{\Phi_{1} \sqcup \Phi_{2}}$ is total, it is enough to show that $N \mathbb{Z}_{\Phi_{1} \sqcup \Phi_{2}} M$. Towards a contradiction, suppose $N \leq_{\Phi_{1}} \sqcup \Phi_{2} M$. Then, by Proposition 30, there are knowledge bases $R_{1}, R_{2}$ such that $M \models$ $\Delta_{R_{1}}\left(\Phi_{1} \sqcup \Phi_{2}\right), N \models \Delta_{R_{2}}\left(\Phi_{1} \sqcup \Phi_{2}\right)$ and $N \models \Delta_{R_{1} \cap R_{2}}\left(\Phi_{1} \sqcup \Phi_{2}\right)$. By (IC5) and Proposition 22, $M \models \Delta_{R_{1}}\left(\Phi_{1}\right), N \models \Delta_{R_{2}}\left(\Phi_{1}\right)$ and $N \models \Delta_{R_{1} \cap R_{2}}\left(\Phi_{1}\right)$. By Proposition 30, we concluded $N \leq_{\Phi_{1}} M$, a contradiction.

(v) Assume that $M<_{\Phi_{1}} \quad N$ and $M<_{\Phi_{2}} N$. We want to show that $M<_{\Phi_{1}} \sqcup \Phi_{2} \quad N$. Since $\leq_{\Phi_{1} \sqcup \Phi_{2}}$ is a total pre-order, it is enough to show that $N \mathbb{Z}_{\Phi_{1} \sqcup \Phi_{2}} M$. Towards a contradiction, suppose $N \leq_{\Phi_{1} \sqcup \Phi_{2}} M$. By Proposition 30, there exist knowledge bases $R_{1}, R_{2}$ such that $M \models$ $\Delta_{R_{1}}\left(\Phi_{1} \sqcup \Phi_{2}\right), N \models \Delta_{R_{2}}\left(\Phi_{1} \sqcup \Phi_{2}\right)$ and $N \models \Delta_{R_{1} \cap R_{2}}\left(\Phi_{1} \sqcup \Phi_{2}\right)$. By (IC5'), each of the three previous conditions give two possibilities. For instance from $M \models \Delta_{R_{1}}\left(\Phi_{1} \sqcup \Phi_{2}\right)$ we get (using Proposition 22) $M \models \Delta_{R_{1}}\left(\Phi_{1}\right)$ or $M \models \Delta_{R_{1}}\left(\Phi_{2}\right)$. Thus, in total, 8 cases can occur. We examine the following 4 cases (the remaining cases can be analyzed in a similar way):

1. $M \models \Delta_{R_{1}}\left(\Phi_{1}\right), N \models \Delta_{R_{2}}\left(\Phi_{1}\right)$ and $N \models \Delta_{R_{1} \cap R_{2}}\left(\Phi_{1}\right)$.

2. $M \models \Delta_{R_{1}}\left(\Phi_{2}\right), N \models \Delta_{R_{2}}\left(\Phi_{2}\right)$ and $N \models \Delta_{R_{1} \cap R_{2}}\left(\Phi_{2}\right)$.

3. $M \models \Delta_{R_{1}}\left(\Phi_{1}\right), N \models \Delta_{R_{2}}\left(\Phi_{2}\right)$ and $N \models \Delta_{R_{1} \cap R_{2}}\left(\Phi_{1}\right)$.

4. $M \models \Delta_{R_{1}}\left(\Phi_{1}\right), N \models \Delta_{R_{2}}\left(\Phi_{2}\right)$ and $N \models \Delta_{R_{1} \cap R_{2}}\left(\Phi_{2}\right)$.

In case 1, by Proposition 30, $N \leq_{\Phi_{1}} M$, a contradiction. In case 2, by Proposition $30, N \leq_{\Phi_{2}} M$, a contradiction. In case 3 , since $M<_{\Phi_{1}} N$ and $N \models \Delta_{R_{1} \cap R_{2}}\left(\Phi_{1}\right)$ by Lemma $32, M \forall R_{1} \cap R_{2}$. This leads to a contradiction, because from $M \models \Delta_{R_{1}}\left(\Phi_{1}\right)$ and the postulate (IC0), we can get $M \models R_{1}$, and therefore $M \models R_{1} \cap R_{2}$. The case 4 leads also to a contradiction. Since $N \models \Delta_{R_{1} \cap R_{2}}\left(\Phi_{2}\right)$ and $M<_{\Phi_{2}} N$ imply, by Lemma $32, M \not \models R_{1} \cap R_{2}$. This contradicts the fact that $M \models R_{1}$, which is a consequence of $M \models \Delta_{R_{1}}\left(\Phi_{1}\right)$ using (IC0). 
(vi) Now we prove that $\leq_{\Phi}$ is smooth. Let $M \models K$, and suppose that $M \notin \min \left(\bmod (K), \leq_{\Phi}\right.$ ). By Lemma $39 M \not \models \Delta_{K}(\Phi)$. Define $\mathcal{H}=\left\{R: M \models \Delta_{R}(\Phi)\right\}$. We claim that for any $R \in \mathcal{H}$, $\Delta_{K}(\Phi) \cup R$ is inconsistent. Towards a contradiction, suppose $\Delta_{K}(\Phi) \cup R$ consistent. Then, by (IC6) and (IC7),

$$
\Delta_{\mathrm{Cn}(K \cup R)}(\Phi)=\operatorname{Cn}\left(\Delta_{K}(\Phi) \cup R\right)
$$

Since $M \models K, \Delta_{R}(\Phi) \cup K$, for any $R \in \mathcal{H}$. By (IC6) and (IC7) we have

$$
\Delta_{\mathrm{Cn}(K \cup R)}(\Phi)=\operatorname{Cn}\left(\Delta_{R}(\Phi) \cup K\right)
$$

From 9 and 10 follows $\operatorname{Cn}\left(\Delta_{R}(\Phi) \cup K\right)=\operatorname{Cn}\left(\Delta_{K}(\Phi) \cup R\right)$. Since $M \models \Delta_{R}(\Phi) \cup K$, we conclude $M \models \Delta_{K}(\Phi)$ which is a contradiction. Thus, we have that $\Delta_{K}(\Phi) \cup R$ is inconsistent. Take $N \models \Delta_{K}(\Phi)$. We know, by Lemma 39, that $N \in \min \left(\bmod (K), \leq_{\Phi}\right)$. Furthermore, by the inconsistency of $\Delta_{K}(\Phi) \cup R$ for any $R \in \mathcal{H}, N \not \models R$ for any $R$ verifying $M \models \Delta_{R}(\Phi)$. Finally, Lemma 32 allows to conclude $N<_{\Phi} M$.

(vii) Assume that $\Delta$ satisfies (Maj). Suppose $M<_{\Phi_{2}} N$. We want to show that there exists $n$ such that $M<_{\Phi_{1} \sqcup \Phi_{2}{ }^{n}} N$. By (Maj) there exists $n$ such that $\Delta_{K}\left(\Phi_{1} \sqcup \Phi_{2}{ }^{n}\right) \supseteq \Delta_{K}\left(\Phi_{2}\right)$ for any

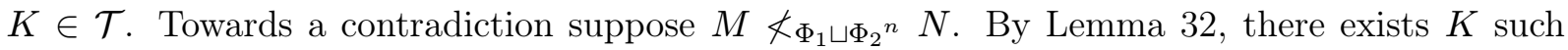
that $N \models \Delta_{K}\left(\Phi_{1} \sqcup \Phi_{2}{ }^{n}\right)$ and $M \models K$. Therefore $N \models \Delta_{K}\left(\Phi_{2}\right)$ and $M \models K$. Again, by Lemma 32, we have $M \nless_{\Phi_{2}} N$, a contradiction.

Now we prove that the assignment $\Phi \mapsto \leq_{\Phi}$ defined starting from $\Delta$, indeed represents $\Delta$. More precisely we have the following:

\section{Proposition 41}

Assume that $\Delta$ satisfies (IC0), (IC1), (IC6) and (IC7). Then $\Delta_{K}(\Phi)=\operatorname{Th}\left(\min \left(\bmod (K), \leq_{\Phi}\right)\right)$

Proof: It is enough to prove that $\bmod \left(\Delta_{K}(\Phi)\right)=\min \left(\bmod (K), \leq_{\Phi}\right)$. First, we note that $\bmod \left(\Delta_{K}(\Phi)\right) \subseteq \min \left(\bmod (K), \leq_{\Phi}\right)$ is exactly what says the Lemma 39 . Now, we prove the reverse inclusion, i.e. $\bmod \left(\Delta_{K}(\Phi)\right) \supseteq \min \left(\bmod (K), \leq_{\Phi}\right)$. Take $M \in \min \left(\bmod (K), \leq_{\Phi}\right)$. The proof of the smoothness property above, says us that $M$ is necessarily in $\Delta_{K}(\Phi)$, otherwise we can find $N=K$ such that $N<_{\Phi} M$ what contradicts the minimality of $M$.

The previous results give us the first half part of Theorem 4. Our next goal is to show the second part of that Theorem, i.e. that a syncretic assignment induces a merging operator defined by the equation 1. Actually we are going to prove a result that give us more precise relationships between the conditions satisfied by the assignment and the postulates satisfied by the operator induced.

\section{Proposition 42}

Let $\Phi \mapsto \leq_{\Phi}$ be an assignment, mapping a knowledge multi-set $\Phi$ in a smooth total preorder $\leq_{\Phi}$. Then the application $\Delta: \mathcal{S} \times \mathcal{T} \rightarrow \mathcal{T}$ defined by $\Delta_{K}(\Phi)=\operatorname{Th}\left(\min \left(\bmod (K), \leq_{\Phi}\right)\right)$ is well defined and satisfies (IC0), (IC1), (IC6) and (IC7). Moreover, the following conditions hold

(i) If the assignment satisfies conditions 1 and 2 of Definition 3 then $\Delta$ satisfies (IC2).

(ii) If the assignment satisfies condition 3 of Definition 3 then $\Delta$ satisfies (IC3). 
(iii) If the assignment satisfies condition 4 of Definition 3 then $\Delta$ satisfies (IC4).

(iv) If the assignment satisfies condition 5 of Definition 3 then $\Delta$ satisfies (IC5).

(v) If the assignment satisfies condition 5' of quasi-syncretic assignment definition then $\Delta$ satisfies $\left(I C 5^{\prime}\right)$.

(vi) If the assignment satisfies condition 7 of majority syncretic assignment definition then $\Delta$ satisfies (Maj).

Proof: Clearly $\Delta$ is well defined. By definition, it follows that $\bmod \left(\Delta_{K}(\Phi)\right) \subseteq \bmod (K)$ therefore $\Delta_{K}(\Phi) \supseteq K$, i.e. (IC0) holds. Note that if $K \forall \perp$ then $\bmod (K) \neq \emptyset$. Thus, by the smoothness of $\leq_{\Phi}$, it follows $\min \left(\bmod (K), \leq_{\Phi}\right) \neq \varnothing$; therefore $\Delta_{K}(\Phi) \nvdash \perp$. Thus, (IC1) holds.

Let us verify (IC6). If $\Delta_{K_{1}}(\Phi) \cup K_{2} \vdash \perp$ then, trivially, $\operatorname{Cn}\left(\Delta_{K_{1}}(\Phi) \cup K_{2}\right) \supseteq \Delta_{K_{1} \cup K_{2}}(\Phi)$. Suppose now that $\Delta_{K_{1}}(\Phi) \cup K_{2} \not \forall \perp$. Let $M \models \Delta_{K_{1}}(\Phi) \cup K_{2}$ then $M \in \min \left(\bmod \left(K_{1}\right), \leq_{\Phi}\right)$ and $M \models K_{2}$. Let $N \models K_{1} \cup K_{2}$, in particular $N \models K_{1}$. So $M \leq_{\Phi} N$. This means that $M \in \min \left(\bmod \left(K_{1} \cup K_{2}\right), \leq_{\Phi}\right)$. Therefore $\operatorname{Cn}\left(\Delta_{K_{1}}(\Phi) \cup K_{2}\right) \supseteq \Delta_{\mathrm{Cn}\left(K_{1} \cup K_{2}\right)}(\Phi)$. This completes the verification of postulate (IC6).

Now, we verify (IC7). Assume that $\Delta_{K_{1}}(\Phi) \cup K_{2}$ is consistent. This together with (IC0) entails the consistency of $K_{1} \cup K_{2}$. Then there exist $N=\Delta_{K_{1}}(\Phi) \cup K_{2}$ and $M=\Delta_{\mathrm{Cn}\left(K_{1} \cup K_{2}\right)}(\Phi)$. We want to show that $M \models \Delta_{K_{1}}(\Phi) \cup K_{2}$. Since $N \models \Delta_{K_{1}}(\Phi) \cup K_{2}$, by (IC0), $N \models K_{1} \cup K_{2}$. So, by minimality of $M$ in $\bmod \left(K_{1} \cup K_{2}\right), M \leq_{\Phi} N$. Also, by (IC0), $M \models K_{1}$. So, by minimality of $N$ in $\bmod \left(K_{1}\right), N \leq_{\Phi} M$. These relations entail $M \simeq_{\Phi} N$. Then $M$ is minimal in $K_{1}$, so, by definition, $M \models \Delta_{K_{1}}(\Phi)$. Note that (IC0) and the fact that $M \models \Delta_{\mathrm{Cn}\left(K_{1} \cup K_{2}\right)}(\Phi)$ entail $M \models K_{2}$. Thus, $M \models \Delta_{K_{1}}(\Phi) \cup K_{2}$.

Next, we are going to prove the assertions (i) to (iv) of the proposition:

(i) We suppose conditions 1 and 2 of Definition 3. Assume that $K \cup(\mathbb{A} \Phi)$ is consistent We want to see that $\Delta_{K}(\Phi)=\operatorname{Cn}(K \cup(\mathbb{A} \Phi))$. Let $M \models K \cup(\mathbb{A} \Phi)$. Since $M \models \mathbb{A} \Phi$, conditions 1 and 2 syncretic assignment, says that $M$ is minimal, in particular, $\left.M \in \min \left(\bmod (K), \leq_{\Phi}\right)\right)$, that is to say $M \models \Delta_{K}(\Phi)$. This proof that $\operatorname{Cn}(K \cup(\mathbb{X} \Phi)) \supseteq \Delta_{K}(\Phi)$ For the inverse inclusion, let us suppose that $M \models \Delta_{K}(\Phi)$; by definition $\left.M \in \min \left(\bmod (K), \leq_{\Phi}\right)\right)$. Towards a contradiction, suppose that $M \not K \cup(\mathbb{A} \Phi)$. Since $M \models K$, necessarily $M \not \mathbb{A} \Phi$. Since $K \cup(\mathbb{X} \Phi)$ is consistent, there is $N$ such that $N \models K \cup(\mathbb{A} \Phi)$. Then, by condition $2, N<_{\Phi} M$. But this, together with $N \models K$, contradicts the fact $\left.M \in \min \left(\bmod (K), \leq_{\Phi}\right)\right)$. Thus $M \models K \cup(\wedge \Phi)$. We have proved $\Delta_{K}(\Phi) \supseteq K \cup(\mathbb{M} \Phi)$.

(ii) Suppose that condition 3 of Definition 3 holds. Suppose that $T \supseteq K, R \supseteq K$ and $\Delta_{K}(T \sqcup R) \cup T \nvdash \perp$. We want to show that $\Delta_{K}(T \sqcup R) \cup R \nvdash \perp$. Let $N=\Delta_{K}(T \sqcup R) \cup T$, then $\left.N \in \min \left(\bmod (K), \leq_{T \sqcup R}\right)\right)$ and $N \models T$ By condition 3 , there exists $M \models R$ such that $M \leq_{T \sqcup R} N$. Since $R \supseteq K$, necessarily $M \models K$. Necessarily $M$ is minimal in $\bmod (K)$ with respect the pre-order $\leq_{T} \sqcup R$. From what follows $\Delta_{K}(T \sqcup R) \cup R \nvdash \perp$.

(iii) Assume that condition 4 of Definition 3 holds. We want to see that (IC4) is satisfied, i.e. $\operatorname{Cn}\left(\Delta_{K}\left(\Phi_{1}\right) \cup \Delta_{K}\left(\Phi_{2}\right)\right) \supseteq \Delta_{K}\left(\Phi_{1} \sqcup \Phi_{2}\right)$. If $\Delta_{K}\left(\Phi_{1}\right) \cup \Delta_{K}\left(\Phi_{2}\right) \vdash \perp$, the verification is trivial. Thus, suppose $\Delta_{K}\left(\Phi_{1}\right) \cup \Delta_{K}\left(\Phi_{2}\right)$ consistent and let $M$ be a valuation such that $M \models \Delta_{K}\left(\Phi_{1}\right) \cup \Delta_{K}\left(\Phi_{2}\right)$. By definition, $M \in \min \left(\bmod (K), \leq_{\Phi_{1}}\right) \cap \min \left(\bmod (K), \leq_{\Phi_{2}}\right)$. In particular, for any $N \in \bmod (K), M \leq_{\Phi_{1}} N$ and $M \leq_{\Phi_{2}} N$. By condition $4, M \leq_{\Phi_{1} \sqcup \Phi_{2}} N$. Thus, $\left.M \in \min \left(\bmod (K), \leq_{\Phi_{1}} \sqcup \Phi_{2}\right)\right)$, i.e. $M \models \Delta_{K}\left(\Phi_{1} \sqcup \Phi_{2}\right)$. Therefore $\operatorname{Cn}\left(\Delta_{K}\left(\Phi_{1}\right) \cup \Delta_{K}\left(\Phi_{2}\right)\right) \supseteq$ 
$\Delta_{K}\left(\Phi_{1} \sqcup \Phi_{2}\right)$.

(iv) Assume that condition 5 of Definition 3 holds. We want to see that (IC5) is satisfied, i.e. if $\Delta_{K}\left(\Phi_{1}\right) \cup \Delta_{K}\left(\Phi_{2}\right)$ is consistent then $\Delta_{K}\left(\Phi_{1} \sqcup \Phi_{2}\right) \supseteq \operatorname{Cn}\left(\Delta_{K}\left(\Phi_{1}\right) \cup \Delta_{K}\left(\Phi_{2}\right)\right)$. By hypothesis, there exists $N$ such that $N \models \Delta_{K}\left(\Phi_{1}\right) \cup \Delta_{K}\left(\Phi_{2}\right)$,. Let $M$ be such that $M \models \Delta_{K}\left(\Phi_{1} \sqcup \Phi_{2}\right)$. We want to show that $M \models \Delta_{K}\left(\Phi_{1}\right) \cup \Delta_{K}\left(\Phi_{2}\right)$. By (IC0), we get easily that $N \models K$; from

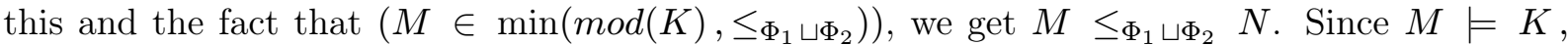
if $M \not \forall \Delta_{K}\left(\Phi_{1}\right)$ then $N<{ }_{\Phi_{1}} M$. By the totality of $\leq_{\Phi_{2}}$ and the fact that $N$ is minimal with respect to this pre-order, we also have $N \leq_{\Phi_{2}} M$ By condition $5, N<_{\Phi_{1} \sqcup \Phi_{2}} M$, a contradiction. Thus, $M \models \Delta_{K}\left(\Phi_{1}\right)$. In an analogous way, we prove that $M \models \Delta_{K}\left(\Phi_{2}\right)$. Therefore $M \models \Delta_{K}\left(\Phi_{1}\right) \cup \Delta_{K}\left(\Phi_{2}\right)$. That is to say $\Delta_{K}\left(\Phi_{1} \sqcup \Phi_{2}\right) \supseteq \operatorname{Cn}\left(\Delta_{K}\left(\Phi_{1}\right) \cup \Delta_{K}\left(\Phi_{2}\right)\right)$.

(v) Assume that condition 5' of syncretic assignment definition holds. We want to see that (IC5') is satisfied, i.e. if $\Delta_{K}\left(\Phi_{1}\right) \cup \Delta_{K}\left(\Phi_{2}\right)$ is consistent then $\Delta_{K}\left(\Phi_{1} \sqcup \Phi_{2}\right) \supseteq\left(\Delta_{K}\left(\Phi_{1}\right) \cap \Delta_{K}\left(\Phi_{2}\right)\right)$. Take $M \models \Delta_{R}\left(\Phi_{1} \sqcup \Phi_{2}\right)$. Towards a contradiction suppose $M \not \Delta_{R}\left(\Phi_{1}\right)$ and $M \not \models \Delta_{R}\left(\Phi_{2}\right)$. Let $P \models \Delta_{R}\left(\Phi_{1}\right) \cup \Delta_{R}\left(\Phi_{2}\right)$, in particular $P \models \Delta_{R}\left(\Phi_{1}\right)$ and $P \models \Delta_{R}\left(\Phi_{2}\right)$. By definition of $\Delta$, $P<_{\Phi_{1}} M$ and $P<_{\Phi_{2}} M$. Then, by $5^{\prime}, P<_{\Phi_{1} \sqcup \Phi_{2}} M$ and by the lemma $32, P \not \models R$. But $P \models \Delta_{R}\left(\Phi_{1}\right)$, so, by (IC0), $P \models R$, a contradiction.

(vi) Assume that condition 7 of majority syncretic assignment definition holds. Given $\Phi_{1}$ and $\Phi_{2}$ we want to show that there exists $n$ such that for any $K, \Delta_{K}\left(\Phi_{1} \sqcup \Phi_{2}{ }^{n}\right) \supseteq \Delta_{K}\left(\Phi_{2}\right)$. As usual, we make a semantical argument based on Proposition 22. Towards a contradiction, suppose $M \not \models \Delta_{K}\left(\Phi_{2}\right)$ and $M \models K$. By smoothness, there exists $N$ such that $N \models \Delta_{K}\left(\Phi_{2}\right)$ and

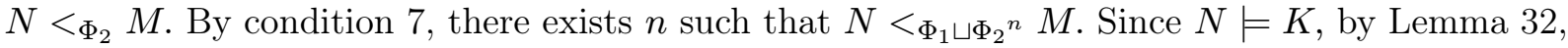
$M \not \models \Delta_{K}\left(\Phi_{1} \sqcup \Phi_{2}{ }^{n}\right)$. Thus, $\Delta_{K}\left(\Phi_{1} \sqcup \Phi_{2}{ }^{n}\right) \supseteq \Delta_{K}\left(\Phi_{2}\right)$.

Putting together Propositions 36, 40, 41 and 42 we obtain Theorems 4, 6 and 8.

Having proved Theorem 4, the task of proving Theorem 10 is reduced to prove Proposition 44 . But before, we state a lemma used in the proof of Proposition 44.

\section{Lemma 43}

Let $\Delta$ a merging operator and $\Phi \mapsto \leq_{\Phi}$ the syncretic assignment given by Theorem 4. Suppose $M \simeq_{\Phi} N, M \neq N, M \models \Delta_{R}(\Phi)$ and $N \models \Delta_{T}(\Phi)$, then for all valuations $P$ and $Q$ if $P \models$ $\Delta_{R}(\Phi) \cap \Delta_{T}(\Phi)$ and $Q \models \Delta_{R}(\Phi) \cap \Delta_{T}(\Phi)$ then $P \simeq_{\Phi} Q$.

Proof: It is a straightforward consequence of the fact that the relation $\simeq_{\Phi}$ is transitive, and the fact $\bmod \left(\Delta_{R}(\Phi) \cap \Delta_{T}(\Phi)\right)=\bmod \left(\Delta_{R}(\Phi)\right) \cup \bmod \left(\Delta_{T}(\Phi)\right)$.

\section{Proposition 44}

Let $\Delta$ a merging operator and $\Phi \mapsto \leq_{\Phi}$ the syncretic assignment given by Theorem 4. Then $\Delta$ satisfies (Arb) iff assignment $\Phi \mapsto \leq_{\Phi}$ satisfies the condition 8 of arbitration syncretic assignment definition.

Proof: (only if) We want to show condition 8. More precisely, we assume $M<_{H_{1}} N, M<_{H_{2}} P$ and $N \simeq_{H_{1} \sqcup H_{2}} P$ and we want to show $M<_{H_{1} \sqcup H_{2}} N$. Take $R_{1}, R_{2}, K_{1}, K_{2}, T_{1}, T_{2}$ such that

1. $M \models \Delta_{R_{1}}\left(H_{1}\right), \Delta_{R_{2}}\left(H_{2}\right)$. 
2. $N \models \Delta_{K_{1}}\left(H_{1}\right), P \models \Delta_{K_{2}}\left(H_{2}\right), K_{1} \cup K_{2} \vdash \perp$ (see the lemma 38)

3. $N \models \Delta_{T_{1}}\left(H_{1} \sqcup H_{2}\right), P \models \Delta_{T_{2}}\left(H_{1} \sqcup H_{2}\right)$.

To realize these conditions it is enough to put $R_{1}=R_{2}=T h(M), K_{1}=T_{1}=T h(N)$ and $K_{2}=T_{2}=T h(P)$. Define

$$
\begin{aligned}
& S_{1}=\Delta_{\mathrm{Cn}\left(R_{1} \cup R_{2}\right)}\left(H_{1} \sqcup H_{2}\right) \cap \mathrm{Cn}\left(\Delta_{K_{1}}\left(H_{1}\right) \cup \Delta_{T_{1}}\left(H_{1} \sqcup H_{2}\right)\right) \\
& S_{2}=\Delta_{\mathrm{Cn}\left(R_{1} \cup R_{2}\right)}\left(H_{1} \sqcup H_{2}\right) \cap \mathrm{Cn}\left(\Delta_{K_{2}}\left(H_{2}\right) \cup \Delta_{T_{2}}\left(H_{1} \sqcup H_{2}\right)\right)
\end{aligned}
$$

We are going to see that $S_{1}, S_{2}$ verify the hypotheses of the postulate of arbitration.

Affirmation 1. $N, P \not \models \Delta_{\mathrm{Cn}\left(R_{1} \cup R_{2}\right)}\left(H_{1} \sqcup H_{2}\right)$.

Proof: Towards a contradiction, suppose $N \models \Delta_{\mathrm{Cn}\left(R_{1} \cup R_{2}\right)}\left(H_{1} \sqcup H_{2}\right)$. By Lemma $37, N \models$ $\Delta_{R_{1}}\left(H_{1}\right)$, which contradicts the fact $M<_{H_{1}} N$. In a similar way, we obtain a contradiction if we suppose that $P \mid=\Delta_{\mathrm{Cn}\left(R_{1} \cup R_{2}\right)}\left(H_{1} \sqcup H_{2}\right)$.

Affirmation $2 N \models S_{1}, P \models S_{2}$.

Proof: This follows straightforward from the facts $N \models \Delta_{K_{1}}\left(H_{1}\right) \cup \Delta_{T_{1}}\left(H_{1} \sqcup H_{2}\right)$ and $P \models$ $\Delta_{K_{2}}\left(H_{2}\right) \cup \Delta_{T_{2}}\left(H_{1} \sqcup H_{2}\right)$.

Affirmation $3 N \not=S_{2}, P \forall S_{1}$.

Proof: Since $K_{1} \cup K_{2} \vdash \perp$,

$$
\operatorname{Cn}\left(\Delta_{K_{1}}\left(H_{1}\right) \cup \Delta_{T_{1}}\left(H_{1} \sqcup H_{2}\right)\right) \cup \operatorname{Cn}\left(\Delta_{K_{2}}\left(H_{2}\right) \cup \Delta_{T_{2}}\left(H_{1} \sqcup H_{2}\right)\right) \vdash \perp .
$$

Since $N \models \Delta_{K_{1}}\left(H_{1}\right) \cup \Delta_{T_{1}}\left(H_{1} \sqcup H_{2}\right)$, we have $N \not \forall \operatorname{Cn}\left(\Delta_{K_{2}}\left(H_{2}\right) \cup \Delta_{T_{2}}\left(H_{1} \sqcup H_{2}\right)\right)$, so, by Affirmation 1 and Proposition 22, necessarily $N \not \models S_{2}$. Similarly we obtain $P \not \models S_{1}$. Therefore

$$
S_{1} \nsubseteq S_{2}, S_{2} \nsubseteq S_{1} \quad(*)
$$

Affirmation $4 \min \left(\bmod \left(S_{1}\right), \leq_{H_{1}}\right)=\bmod \left(\Delta_{\mathrm{Cn}\left(R_{1} \cup R_{2}\right)}\left(H_{1} \sqcup H_{2}\right)\right)$.

From the definition of $R_{1}, R_{2}, K_{1}$ and $T_{1}$ it is easy to see that $\bmod \left(S_{1}\right)=\{M, N\}$. The fact $M<_{H_{1}} N$ says us that $\min \left(\bmod \left(S_{1}\right), \leq_{H_{1}}\right)=\{M\}$. On the other hand, it is easy to see that $\bmod \left(\Delta_{\mathrm{Cn}\left(R_{1} \cup R_{2}\right)}\left(H_{1} \sqcup H_{2}\right)\right)=\{M\}$. Therefore $\min \left(\bmod \left(S_{1}\right), \leq_{H_{1}}\right)=\bmod \left(\Delta_{\mathrm{Cn}\left(R_{1} \cup R_{2}\right)}\left(H_{1} \sqcup H_{2}\right)\right)$.

With an analogous argument we can prove the following:

Affirmation $5 \min \left(\bmod \left(S_{2}\right), \leq_{H_{2}}\right)=\bmod \left(\Delta_{\mathrm{Cn}\left(R_{1} \cup R_{2}\right)}\left(H_{1} \sqcup H_{2}\right)\right)$.

From affirmations 4 and 5 , we get

$$
\Delta_{S_{1}}\left(H_{1}\right)=\Delta_{S_{2}}\left(H_{2}\right)=\Delta_{\mathrm{Cn}\left(R_{1} \cup R_{2}\right)}\left(H_{1} \sqcup H_{2}\right) \quad(* *)
$$

From $\operatorname{Cn}\left(\Delta_{K_{1}}\left(H_{1}\right) \cup \Delta_{T_{1}}\left(H_{1} \sqcup H_{2}\right)\right) \cup \mathrm{Cn}\left(\Delta_{K_{2}}\left(H_{2}\right) \cup \Delta_{T_{2}}\left(H_{1} \sqcup H_{2}\right)\right) \vdash \perp$, we get $\bmod \left(\Delta_{K_{1}}\left(H_{1}\right) \cup \Delta_{T_{1}}\left(H_{1} \sqcup H_{2}\right)\right) \cap \bmod \left(\Delta_{K_{2}}\left(H_{2}\right) \cup \Delta_{T_{2}}\left(H_{1} \sqcup H_{2}\right)\right)=\varnothing$.

In the proof of affirmation 4 we have seen that $\Delta_{R_{1}}\left(H_{1}\right) \cup \Delta_{K_{1}}\left(H_{1}\right) \vdash \perp$. Similarly, one has that $\Delta_{R_{2}}\left(H_{2}\right) \cup \Delta_{K_{2}}\left(H_{2}\right) \vdash \perp$

Thus

$$
\begin{aligned}
& \bmod \left(S_{1}\right) \backslash \bmod \left(S_{2}\right)=\bmod \left(\Delta_{K_{1}}\left(H_{1}\right) \cup \Delta_{T_{1}}\left(H_{1} \sqcup H_{2}\right)\right) \\
& \bmod \left(S_{2}\right) \backslash \bmod \left(S_{1}\right)=\bmod \left(\Delta_{K_{2}}\left(H_{2}\right) \cup \Delta_{T_{2}}\left(H_{1} \sqcup H_{2}\right)\right)
\end{aligned}
$$


By definition, $S_{1} \diamond S_{2}=\operatorname{Th}\left(\bmod \left(S_{1}\right) \triangle \bmod \left(S_{2}\right)\right)$, so,

$$
\begin{aligned}
S_{1} \diamond S_{2} & =\operatorname{Th}\left(\bmod \left(\Delta_{K_{1}}\left(H_{1}\right) \cup \Delta_{T_{1}}\left(H_{1} \sqcup H_{2}\right)\right) \cup \bmod \left(\Delta_{K_{2}}\left(H_{2}\right) \cup \Delta_{T_{2}}\left(H_{1} \sqcup H_{2}\right)\right)\right) \\
& =\operatorname{Th}\left(\bmod \left(\left(\Delta_{K_{1}}\left(H_{1}\right) \cup \Delta_{T_{1}}\left(H_{1} \sqcup H_{2}\right)\right) \cap\left(\Delta_{K_{2}}\left(H_{2}\right) \cup \Delta_{T_{2}}\left(H_{1} \sqcup H_{2}\right)\right)\right)\right)
\end{aligned}
$$

Affirmation 6 If $\left.Q_{1} \models \Delta_{T_{1}}\left(H_{1} \sqcup H_{2}\right)\right), Q_{2} \models \Delta_{T_{2}}\left(H_{1} \sqcup H_{2}\right)$ then $Q_{1} \simeq_{H_{1} \sqcup H_{2}} Q_{2}$.

Proof: Use the facts $N \simeq_{H_{1} \sqcup H_{2}} P, N \models \Delta_{T_{1}}\left(H_{1} \sqcup H_{2}\right)$ ) and $P \models \Delta_{T_{2}}\left(H_{1} \sqcup H_{2}\right)$ and the transitivity of $\simeq H_{1} \sqcup H_{2}$.

Since

$$
\begin{aligned}
& \bmod \left(\left(\Delta_{K_{1}}\left(H_{1}\right) \cup \Delta_{T_{1}}\left(H_{1} \sqcup H_{2}\right)\right) \subseteq \bmod \left(\Delta_{T_{1}}\left(H_{1} \sqcup H_{2}\right)\right)\right. \\
& \left.\bmod \left(\Delta_{K_{2}}\left(H_{2}\right) \cup \Delta_{T_{2}}\left(H_{1} \sqcup H_{2}\right)\right)\right) \subseteq \bmod \left(\Delta_{T_{2}}\left(H_{1} \sqcup H_{2}\right)\right)
\end{aligned}
$$

we can assure

$$
\begin{aligned}
\bmod \left(\left(\Delta_{K_{1}}\left(H_{1}\right) \cup \Delta_{T_{1}}\right.\right. & \left.\left.\left(H_{1} \sqcup H_{2}\right)\right) \cap\left(\Delta_{K_{2}}\left(H_{2}\right) \cup \Delta_{T_{2}}\left(H_{1} \sqcup H_{2}\right)\right)\right) \\
& \subseteq \bmod \left(\Delta_{T_{1}}\left(H_{1} \sqcup H_{2}\right) \cap \Delta_{T_{2}}\left(H_{1} \sqcup H_{2}\right)\right)
\end{aligned}
$$

Thus, the models of $S_{1} \diamond S_{2}$ are in the same level regarding the order $\leq_{H_{1} \sqcup H_{2}}$, and therefore $\min \left(\bmod \left(S_{1} \diamond S_{2}\right), \leq_{H_{1}} \sqcup H_{2}\right)=\bmod \left(S_{1} \diamond S_{2}\right)$. That is to say

$$
\Delta_{S_{1} \diamond S_{2}}\left(H_{1} \sqcup H_{2}\right)=S_{1} \diamond S_{2} \quad(* * *)
$$

The conditions $(*),(* *),(* * *)$ say that the theories $S_{1}, S_{2}$ verify the hypotheses of the arbitration postulate, and therefore

$$
\Delta_{S_{1} \cap S_{2}}\left(H_{1} \sqcup H_{2}\right)=\Delta_{S_{1}}\left(H_{1}\right)
$$

By (**) we have that $M \models \Delta_{S_{1}}\left(H_{1}\right)$, also $N \not \Delta_{S_{1}}\left(H_{1}\right)$, to be $M<_{H_{1}} N$. By equation 11 , $M \models \Delta_{S_{1} \cap S_{2}}\left(H_{1} \sqcup H_{2}\right), N \not \Delta_{S_{1} \cap S_{2}}\left(H_{1} \sqcup H_{2}\right)$. Since $N \models S_{1}, N \models S_{1} \cap S_{2}$. This means that $N$ is a model of $S_{1} \cap S_{2}$ which is not minimal for the pre-order $\leq_{H_{1} \sqcup H_{2}}$. Since $M$ is a minimal model of $S_{1} \cap S_{2}$ for the pre-order $\leq_{H_{1} \sqcup H_{2}}$, necessarily $M<_{H_{1} \sqcup H_{2}} N$.

(if) Now suppose that $\Phi \mapsto \leq_{\Phi}$ is an arbitration syncretic assignment, then we are going to prove that $\Delta$ satisfies (Arb). In order to do that, suppose that we have two theories $K_{1}, K_{2}$, such that

$$
\begin{aligned}
& K_{1} \nsubseteq K_{2}, \quad K_{2} \nsubseteq K_{1}, \\
& \Delta_{K_{1}}\left(H_{1}\right)=\Delta_{K_{2}}\left(H_{2}\right), \\
& \Delta_{K_{1} \diamond K_{2}}\left(H_{1} \sqcup H_{2}\right)=K_{1} \diamond K_{2}
\end{aligned}
$$

We want to show that $\Delta_{K_{1} \cap K_{2}}\left(H_{1} \sqcup H_{2}\right)=\Delta_{K_{1}}\left(H_{1}\right)$. First, we will prove that $\Delta_{K_{1} \cap K_{2}}\left(H_{1} \sqcup H_{2}\right) \supseteq$ $\Delta_{K_{1}}\left(H_{1}\right)$ Towards a contradiction, suppose that there exists $N$ such that $N \models \Delta_{K_{1} \cap K_{2}}\left(H_{1} \sqcup H_{2}\right)$ such that $N \not \Delta_{K_{1}}\left(H_{1}\right)$.

By $(\mathrm{IC} 0), N \models K_{1} \cap K_{2}$. Thus, $N \in \bmod \left(K_{1}\right) \cup \bmod \left(K_{2}\right)$. There are three cases to examine: 
1. $N \in \bmod \left(K_{1}\right) \cap \bmod \left(K_{2}\right)$.

2. $N \in \bmod \left(K_{1}\right), \quad N \notin \bmod \left(K_{2}\right)$.

3. $N \notin \bmod \left(K_{1}\right), \quad N \in \bmod \left(K_{2}\right)$.

The cases 2 and 3 they are symmetrical. Thus, we will only study the first two cases.

Case 1).- $N \models K_{1} \quad N \models K_{2}$. By the hypotheses, there is $M$ such that $M \models \Delta_{K_{1}}\left(H_{1}\right)=$ $\Delta_{K_{2}}\left(H_{2}\right)$. This and the fact $N \not \models \Delta_{K_{1}}\left(H_{1}\right)$ entail $M<_{H_{1}} N$ and $M<_{H_{2}} N$. By condition 5 of the arbitration syncretic assignment, we get $M<_{H_{1} \sqcup H_{2}} N$. But $M \models K_{1} \cap K_{2}$ and $N \models \Delta_{K_{1} \cap K_{2}}\left(H_{1} \sqcup H_{2}\right)$ says, by Lemma $32, M \nless_{H_{1} \sqcup H_{2}} N$, a contradiction.

Case 2).- Suppose that $N \models K_{1}$ and $N \not \models K_{2}$. Since $K_{1} \nsubseteq \subset K_{2}$, there is $P \models K_{2}$ such that $P \not \models$ $K_{1}$. Since $\Delta_{K_{1}}\left(H_{1}\right)=\Delta_{K_{2}}\left(H_{2}\right)$, we have that neither $N$ neither $P$ are models of $\Delta_{K_{1}}\left(H_{1}\right)=$ $\Delta_{K_{2}}\left(H_{2}\right)$. For any $M=\Delta_{K_{1}}\left(H_{1}\right)$ we have $M<_{H_{1}} N$ and $M<_{H_{2}} P$. It is clear that $N, P \models$ $K_{1} \diamond K_{2}$, because $N, M \in \bmod \left(K_{1}\right) \triangle \bmod \left(K_{2}\right)$. Since $\Delta_{K_{1} \diamond K_{2}}\left(H_{1} \sqcup H_{2}\right)=K_{1} \diamond K_{2}$, necessarily $N, P \models \Delta_{K_{1} \diamond K_{2}}\left(H_{1} \sqcup H_{2}\right)$. Moreover $N \simeq_{H_{1} \sqcup H_{2}} P$. Condition 8 of the arbitration syncretic assignment leads to $M<_{H_{1} \sqcup H_{2}} N$. But $N=\Delta_{K_{1} \cap K_{2}}\left(H_{1} \sqcup H_{2}\right)$ and $M \models K_{1} \cap K_{2}$ entail, by Lemma 32, $M \nless_{H_{1} \sqcup H_{2}} N$, a contradiction.

To finish the proof, it remains to see that $\Delta_{K_{1}}\left(H_{1}\right) \supseteq \Delta_{K_{1} \cap K_{2}}\left(H_{1} \sqcup H_{2}\right)$.

Affirmation 7 If $M \models \Delta_{K_{1}}\left(H_{1}\right), N \models K_{1}, N \not \models K_{2}$, then $M<_{H_{1}} N$.

Proof: Towards a contradiction suppose $N \leq_{H_{1}} M$. Then, by Theorem $4, M \in \min \left(\bmod \left(K_{1}\right), \leq_{H_{1}}\right.$ ) and therefore $N$ is a minimal model of $K_{1}$ with respect to $\leq_{H_{1}}$. So, $N \models \Delta_{K_{1}}\left(H_{1}\right)$ and, by hypothesis, we have that $N \models \Delta_{K_{2}}\left(H_{2}\right)$. (IC0) entails $N \models K_{2}$, a contradiction.

Affirmation 8 If $M \models \Delta_{K_{1}}\left(H_{1}\right), N^{\prime} \not \models K_{1}, N^{\prime} \models K_{2}$, then $M<_{H_{2}} N^{\prime}$.

Proof: Analogous to the the proof of previous affirmation.

Now take $N, N^{\prime} \in \bmod \left(K_{1} \diamond K_{2}\right)$. Since $\Delta_{K_{1} \diamond K_{2}}\left(H_{1} \sqcup H_{2}\right)=K_{1} \diamond K_{2}$, necessarily $N \simeq_{H_{1} \sqcup H_{2}} N^{\prime}$. Take any $M \models \Delta_{K_{1}}\left(H_{1}\right)$. Affirmations 7 and 8 , and the condition 8 of arbitration syncretic assignment entail $M<_{H_{1} \sqcup H_{2}} N$.

Now suppose that $N \models K_{1} \cup K_{2}$, that is $N \in \bmod \left(K_{1}\right) \cap \bmod \left(K_{2}\right)$. If $M \models \Delta_{K_{1}}\left(H_{1}\right)=$ $\Delta_{K_{2}}\left(H_{2}\right)$, then $M \leq_{H_{1}} N$ and $M \leq_{H_{2}} N$. By condition 4 of the syncretic assignment, we have $M \leq_{H_{1} \sqcup H_{2}} N$. Now, observe that $\bmod \left(K_{1} \cap K_{2}\right)=\bmod \left(K_{1} \cup K_{2}\right) \cup \bmod \left(K_{1} \diamond K_{2}\right)$. Therefore, any model $M$ of $\Delta_{K_{1}}\left(H_{1}\right)$ is a minimal model of $K_{1} \cap K_{2}$ with respect to pre-order $\leq_{H_{1} \sqcup H_{2}}$, i.e. $M$ is a model of $\Delta_{K_{1} \cap K_{2}}\left(H_{1} \sqcup H_{2}\right)$.

As a straightforward corollary of previous proposition we have Theorem 10.

Now, we are going to prove Proposition 13. We prove the part (i). Part (ii) is proved in a similar way. We begin with the following observation, the proof of which is trivial.

\section{Observation 45}

$d_{\Sigma}\left(M, \Phi_{1} \sqcup \Phi_{2}\right)=d_{\Sigma}\left(M, \Phi_{1}\right)+d_{\Sigma}\left(M, \Phi_{2}\right)$

\section{Proposition 46}


$\Phi \mapsto \leq_{\Phi}^{\Sigma}$ is a majority syncretic assignment.

Proof: $\leq$ is reflexive, transitive and total over $\overline{\mathbb{R}^{+}}$. This entails $\leq_{\Phi}^{\Sigma}$ is reflexive, transitive and total, i.e. $\leq_{\Phi}^{\Sigma}$ is a total pre-order. When $d_{\Sigma}(M, \Phi)=d_{\Sigma}(N, \Phi)$ we write $M \simeq_{\Phi}^{\Sigma} N$. Now we check that $\Phi \mapsto \leq_{\Phi}^{\Sigma}$ verify the conditions of a majority syncretic assignment.

1. Suppose $M \models \mathbb{A} \Phi, N \models \mathbb{A} \Phi$, then $M \models K, \forall K \in \Phi$ and $N \models K, \forall K \in \Phi$. Therefore, $d(M, K)=0, \forall K \in \Phi$ and $d(N, K)=0, \forall K \in \Phi$. From this follows $d_{\Sigma}(M, \Phi)=0, d_{\Sigma}(N, \Phi)=0$. So, $d_{\Sigma}(M, \Phi)=d_{\Sigma}(N, \Phi)$, i.e. $M \simeq_{\Phi}^{\Sigma} N$.

2. Suppose $M \models \mathbb{A} \Phi, N \not \forall \mathbb{\wedge} \Phi$, then there exists $K \in \Phi$ such that $N \not \models K$. Therefore $d_{\Sigma}(N, K)>0$ and $d_{\Sigma}(M, \Phi)<d_{\Sigma}(N, \Phi)$. Thus $M<_{\Phi}^{\Sigma} N$.

3. Let $M \models K$, so, $d(M, K)=0$. Since $d(M, T)=\inf \{d(M, N): N \models T\}$, by condition 3 of pseudo-distance, there exists $N \models T$ such that $d(M, T)=d(M, N)$. But $d(N, K)=$ $\inf \left\{d\left(M^{\prime}, N\right): M^{\prime} \models K\right\} \leq d(M, N)$. Thus, by Observation 45, $d_{\Sigma}(N, K \sqcup T)=d_{\Sigma}(N, K)+$ $d_{\Sigma}(N, T)$. So, $d_{\Sigma}(N, K \sqcup T)=d_{\Sigma}(N, K) \leq d_{\Sigma}(M, N)=d_{\Sigma}(M, K \sqcup T)$, this implies $N \leq_{K \sqcup T}^{\Sigma} M$.

4. Suppose $M \leq_{\Phi_{1}}^{\Sigma} N$ and $M \leq_{\Phi_{2}}^{\Sigma} N$. By definition, $d_{\Sigma}\left(M, \Phi_{1}\right) \leq d_{\Sigma}\left(N, \Phi_{1}\right)$ and $d_{\Sigma}\left(M, \Phi_{2}\right) \leq d_{\Sigma}\left(N, \Phi_{2}\right)$. Thus $d_{\Sigma}\left(M, \Phi_{1}\right)+d_{\Sigma}\left(M, \Phi_{2}\right) \leq d_{\Sigma}\left(N, \Phi_{1}\right)+d_{\Sigma}\left(N, \Phi_{2}\right)$. By Observation $45, M \leq \leq_{\Phi_{1} \sqcup \Phi_{2}}^{\Sigma} N$.

5. Suppose $M<\sum_{\Phi_{1}}^{\Sigma} N, M \leq_{\Phi_{2}}^{\Sigma} N$. An argument similar to previous one, but using the strict monotony of the sum instead of large monotony, leads to $M<_{\Phi_{1}}^{\Sigma} \sqcup \Phi_{2} N$.

6. We want to show that $\leq_{\Phi}^{\Sigma}$ is smooth. Suppose $M \models K$ and $M$ is not a minimal model of $K$ with respect to pre-order $\leq_{\Phi}^{\Sigma}$. The set $\left\{d_{\Sigma}(P, \Phi): P \mid K\right\}$ is contained in $\overline{\mathbb{R}^{+}}$therefore it has an great lower bound (infimum). Take $N=K$ such that $d_{\Sigma}(N, \Phi) \leq d_{\Sigma}(P, \Phi)$ for any $P \models K$ (the existence of a such $N$ is guaranteed by condition 3 of pseudo-distance). By definition of $\leq_{\Phi}^{\Sigma}$, necessarily $N$ is a minimal model of $K$ with respect to $\leq_{\Phi}^{\Sigma}$. Furthermore, $N<\sum_{\Phi}^{\Sigma} M$.

7. Suppose $M<_{\Phi_{2}}^{\Sigma} N$. We want to show that there exist $n$ such that $M<\Phi_{1} \sqcup \Phi_{2}^{n} N$. By the hypotheses we have $d_{\Sigma}\left(M, \Phi_{2}\right)<d_{\Sigma}\left(N, \Phi_{2}\right)$. By the Archimedean property of $\mathbb{R}$ there exists $n$ such that

$$
n \cdot\left(\left(d_{\Sigma}\left(N, \Phi_{2}\right)-d_{\Sigma}\left(M, \Phi_{2}\right)\right)>d_{\Sigma}\left(M, \Phi_{1}\right)-d_{\Sigma}\left(N, \Phi_{2}\right)\right.
$$

Therefore

$$
d_{\Sigma}\left(M, \Phi_{1}\right)+n \cdot d_{\Sigma}\left(M, \Phi_{2}\right)<d_{\Sigma}\left(N, \Phi_{2}\right)+n \cdot d_{\Sigma}\left(N, \Phi_{2}\right)
$$

Finally by Observation 45, we have

$$
d_{\Sigma}\left(M, \Phi_{1} \sqcup \Phi_{2}^{n}\right)<d_{\Sigma}\left(N, \Phi_{1} \sqcup \Phi_{2}^{n}\right)
$$

The proofs that $\Phi \mapsto \leq_{\Phi}^{\max }$ and $\Phi \mapsto \leq_{\Phi}^{G \max }$ are quasi-syncretic and arbitration assignments respectively are left as an exercise for the reader. 
We finish this section of proofs with the proof of Theorem 15.

Proof of Theorem 15: We want to show that if $T \supseteq R$ then there exists $n$ such that $\left(\Delta_{R}^{\Sigma}\right)^{n}(\Phi, T) \supseteq T$. First we prove the following claim:

Claim.- Assume that $S \supseteq R$ and $T \supseteq R$. We are going to prove that there exists $n$ such that $\left(\Delta_{R}^{\Sigma}\right)^{n}(S \sqcup T) \supseteq T$.

Proof of the claim: Put $a$ the distance between $S$ and $T$, i.e. $d(S, T)=a$. First suppose that $a=\infty$. This means that for any $M$ and $N$ such that $M \models S, N \models T, d(M, N)=\infty$. For any $P \models R$ there are $M$ and $N$ such that $M \models S, N \models T, d(P, S)=d(P, M)$ and $d(P, T)=d(P, N)$. But, using the Triangle Inequality, we have $d_{\Sigma}(P, S \sqcup T)=d(P, S)+d(P, T)=d(P, M)+d(P, N) \geq$ $d(M, N)=\infty$. Thus, $d_{\Sigma}(P, S \sqcup T)=\infty$. Therefore any model of $R$ is minimal with respect to preorder $\leq_{S \sqcup T}^{\Sigma}$. By definition, $\Delta_{R}^{\Sigma}(S \sqcup T)=R$. Since $\Delta_{R}^{\Sigma^{2}}(S, T)=\Delta_{R}^{\Sigma}\left(\Delta_{R}^{\Sigma}(S \sqcup T) \sqcup T\right)=\Delta_{R}^{\Sigma}(R \sqcup T)$, by $(\mathrm{IC} 2), \Delta_{R}^{\Sigma^{2}}(S, T)=\operatorname{Cn}(R \cup T)$. In particular $\Delta_{R}^{\Sigma^{2}}(S, T) \supseteq T$.

Now suppose that $a<\infty$. By the property 3 of pseudo-distances there are valuations $M$ and $N$ such that $M \models S, N \models T$, and $d(M, N)=a$, so, $d(M, N)=d(M, T)=d(N, S)=a$. We claim that $\min \left\{d_{\Sigma}(P, S \sqcup T): P \models R\right\}=a$. By hypothesis $T \supseteq R$, so $N \models R$. Since $d_{\Sigma}(N, S \sqcup T)=d(N, S)+d(N, T)$, and $d(N, T)=0$, necessarily $d_{\Sigma}(N, S \sqcup T)=d(N, S)=a$. Therefore $\min \left\{d_{\Sigma}(P, S \sqcup T): P \mid R\right\} \leq a$. But, using the Triangle Inequality $d_{\Sigma}(P, S \sqcup T)=$ $d(P, S)+d(P, T)=d\left(P, M^{\prime}\right)+d\left(P, N^{\prime}\right) \geq d\left(M^{\prime}, N^{\prime}\right) \geq a$. So, $\min \left\{d_{\Sigma}(P, S \sqcup T): P \models R\right\}=a$. Since $N$ verifies $d_{\Sigma}(N, S \sqcup T)=a$, necessarily $N$ is a minimal model of $R$ with respect to preorder $\leq_{S \sqcup T}^{\Sigma}$. By definition, $N \models \Delta_{R}^{\Sigma}(S \sqcup T)$, in particular $\left(\Delta_{R}^{\Sigma}(S \sqcup T) \cup T\right) \cup R$ is consistent. By (IC2), $\Delta_{R}^{\Sigma}\left(\Delta_{R}^{\Sigma}(S \sqcup T) \sqcup T\right)=\operatorname{Cn}\left(\Delta_{R}^{\Sigma}(S \sqcup T) \cup T \cup R\right)=\operatorname{Cn}\left(\Delta_{R}^{\Sigma}(S \sqcup T) \cup T\right)$. In particular $\Delta_{R}^{\Sigma}\left(\Delta_{R}^{\Sigma}(S \sqcup T) \sqcup T\right) \supseteq T$. This finish the proof of the claim.

In order to finish the proof of the proposition, put $S=\Delta_{R}^{\Sigma}(\Phi \sqcup T)$. By the claim we get $\left(\Delta_{R}^{\Sigma}\right)^{3}(\Phi, T) \supseteq T$.

\section{References}

[1] C. E. Alchourrón, P. Gärdenfors, and D. Makinson: On The Logic Of Theory Change: Partial Meet Contraction And Revision Functions. Journal of Symbolic Logic, 50:510-530, 1985.

[2] C. Baral, S. Kraus, J. Minker, and V. S. Subrahmanian. Combining knowledge bases consisting of first-order theories. Computational Intelligence, 8(1):45-71, 1992.

[3] S. Benferhat, S. Konieczny, O. Papini and R. Pino Pérez. Iterated revision by epistemic states: axioms, semantics and syntax. In Proceedings of the Fourteenth European Conference on Artificial Intelligence, ECAI'2000. Berlin, Germany. August 20-25, 2000, pp 13-17.

[4] L. Cholvy and T. Hunter. Fusion in logic: a brief overview. In Proceedings of the Fourth European Conference on Symbolic and Quantitative Approaches to Reasoning with Uncertainty (ECSQARU'97), Lecture Notes in Computer Science 1244, pages 86-95, 1997. 
[5] L. Cholvy. Reasoning about merged information. In D. M. Gabbay and Ph. Smets, editors, Handbook of Defeasible Reasoning and Uncertainty Management Systems, volume 3, pages 233-263. Kluwer Academic Publishers, 1998.

[6] M. Dalal. Updates in propositional databases, Thecnical report, Rutgers University, 1988.

[7] P. Gärdenfors. Belief revision and nonmonotonoc logic: two sides of the same coin? In Proceedings of the Ninth European Conference on Artificial Intelligence (ECAI'90), 1990, pp. $768-773$.

[8] P. Gärdenfors: Knowledge in Flux: Modeling the Dynamics of Epistemic States, MIT Press, Cambridge, USA, 1988.

[9] H. Katsuno, A. O. Mendelzon. Propositional knowledge base revision and minimal change. Artificial Intelligence, 52:263-294, 1991.

[10] S. Konieczny: Sur la Logique du Changement: Révision et Fusion de bases de Connaissanse. PhD Thesis. Université de Lille. 1999.

[11] S. Konieczny and R. Pino Pérez. On the logic of merging. In Proceedings of the Sixth International Conference on Principles of Knowledge Representation and Reasoning (KR'98), Trento, Italy, June 2-5, 1998, pp. 488-498.

[12] S. Konieczny and R. Pino Pérez. Merging with Integrity Constraints In Proceedings of the Fifth European Conference on Symbolic and Quantitative Approaches to Reasoning with Uncertainty (ECSQARU'99), Lecure Notes in Artificial Intelligence 1638, July 2-9, 1999, pp. 233-244,

[13] S. Konieczny and R. Pino Pérez. A framework for iterated revision. Journal of Applied and Non Classical Logics, vol 10, number 3-4, 339-367, 2000.

[14] S. Konieczny and R. Pino Pérez. Sur la représentation des états épistémiques et la révision iterée. In Révision des croyances. Pierre Livet, Ed. pp 181-202. Hermes-Lavoisier, Paris. 2002 .

[15] S. Konieczny and R. Pino Pérez. Merging information under constraints: a logical framework Journal of Logic and Computation, 12(5): 773-808, 2002.

[16] S. Konieczny and R. Pino Pérez. Propositional Belief Base Merging or how to Merge Beliefs/Goals coming from several Sources and some links with Social Choice Theory. European Journal of Operational Research. To appear.

[17] S. Kraus, D. Lehmann and M. Magidor. Nonmonotonic reasoning, preferential models and cumulative logics. Artificial Intelligence, 44:167-207, 1990.

[18] P. Liberatore and M. Schaerf. Arbitration (or how to merge knowledge bases). IEEE Transactions on Knowledge and Data Engineering, 10(1):76-90, 1998.

[19] J. Lin and A. O. Mendelzon. Knowledge base merging by majority. In Dynamic Worlds: From the Frame Problem to Knowledge Management. Kluwer, 1999. 
[20] E. Pacuit and S. Salame. Majority Logic. In Proceedings of the Ninth International Conference on Principles of Knowledge Representation and Reasoning (KR'04), Whistler, Canada, June 2-5, 2004, pp. 598-605.

[21] R. Pino Pérez, C. Uzcátegui: On the Representation Theorems for nonmonotonic consequence relations. Journal of Symbolic Logic, 65(3):1321-1337, 2000.

[22] P. Z. Revesz. On the semantics of theory change: arbitration between old and new information. In Proceedings of the $12^{\text {th }}$ ACM SIGACT-SIGMOD-SIGART Symposium on Principles of Databases, 1993, pp. 71-92.

[23] P. Z. Revesz. On the semantics of arbitration. International Journal of Algebra and Computation, 7(2):133-160, 1997.

José Luis Chacón and Ramón Pino Pérez

Departamento de Matemáticas

Facultad de Ciencias

Universidad de Los Andes

Mérida, Venezuela

\{jlchacon, pino\}@ula.ve

* Corresponding author information:

Phone: (58) 2742401306 or (58) 4166741139

Fax: (58) 2742401345 or (58) 2742401345

E-mail: pino@ula.ve

Postal address: Departamento de Matemáticas, Facultad de Ciencias, Universidad de Los Andes, La Hechicera, Mérida 5101, Venezuela 